\title{
Investigation of the Neurovascular Coupling in Positive and Negative BOLD Responses in Human Brain at 7T
}

Laurentius Huber $^{a^{*}}$, J ozien Goense ${ }^{b, c}$, Aneurin J . Kennerley ${ }^{d}$, Dimo Ivanov ${ }^{e}$, Steffen N. Krieger $^{a, f}$, J öran Lepsien ${ }^{a}$, Robert Trampel ${ }^{a}$, Robert Turner ${ }^{a}$, and Harald E. Möller ${ }^{a}$

a Max Planck Institute for Human Cognitive and Brain Sciences Leipzig, Germany

${ }^{b}$ Max Planck Institute for Biological Cybernetics, Tübingen, Germany

c Institute of Neuroscience and Psychology, University of Glasgow, Glasgow, UK

d Signal Processing in Neuroimaging and Systems Neuroscience, University of Sheffield, UK

e Maastricht Brain Imaging Centre, Maastricht University, Maastricht, The Netherlands

${ }^{f}$ Monash Biomedical Imaging, Monash University, Melbourne, Victoria, Australia

*Corresponding author:

Laurentius Huber

PhD-student, NMR Unit, Max Planck Institute for Human Cognitive and Brain Sciences

Stephanstraße 1A, 04103 Leipzig, Germany

Email: Ihuber@cbs.mpg.de

Phone: +49 341 9940-2427

Running Title: Features of BOLD and CBV during Excitation and Inhibition

The body of the text contains 7600 words

Highlights: (3-5 Bullet-Points with max. 85 characters including spaces)

- We developed an MRI-method estimating arterial/venous CBV and BOLD signal changes.

- Hemodynamics of excitation and inhibition was investigated in human brain at 7T.

- We found different timecourses, layer-dependence and arterio-venous interaction.

- Our results suggest different neurovascular coupling for excitation and inhibition.

\begin{abstract}
Decreases in stimulus-dependent blood oxygenation level dependent (BOLD) signal and their underlying neurovascular origins have recently gained considerable interest. In this study a multiecho, BOLD-corrected vascular space occupancy (VASO) functional magnetic resonance imaging ( $\mathrm{FMRI}$ ) technique was used to investigate neurovascular responses during stimuli that elicit positive and negative BOLD responses in human brain at 7T. Stimulus-induced BOLD, cerebral blood volume (CBV), and cerebral blood flow (CBF) changes were measured and analyzed in 'arterial' and 'venous' blood compartments in macro- and microvasculature. We found that the overall interplay of mean $\mathrm{CBV}, \mathrm{CBF}$ and BOLD responses is similar for tasks inducing positive and negative BOLD responses. Some aspects of the neurovascular coupling however, such as the temporal response, cortical depth dependence, and the weighting between 'arterial' and 'venous' contributions, are significantly different for the different task conditions. Namely, while for excitatory tasks the BOLD response peaks at the cortical surface, and the CBV change is similar in cortex and pial vasculature, inhibitory tasks are associated with a maximum negative BOLD response in deeper layers, with CBV showing strong constriction of surface arteries and a faster return to baseline. The different interplay of CBV, $\mathrm{CBF}$ and BOLD during excitatory and inhibitory responses suggests different underlying hemodynamic mechanisms.
\end{abstract}


Abbreviations: $\mathrm{BOLD}=$ blood oxygenation level dependent; $\mathrm{CBF}=$ cerebral blood flow; $\mathrm{CBV}=$ cerebral blood volume; $\mathrm{CBV}_{\mathrm{a}}=$ 'arterial' $\mathrm{CBV} ; \mathrm{CBV}_{\text {tot }}=$ total $\mathrm{CBV} ; \mathrm{CBV}_{\mathrm{v}}=$ 'venous' $\mathrm{CBV} ; \mathrm{CMRO}_{2}=$ cerebral metabolic rate of $\mathrm{O}_{2} ; \mathrm{CNR}=$ contrast to noise ratio; $\mathrm{CSF}=$ cerebrospinal fluid; $\triangle \mathrm{CBV}=$ change in $\mathrm{CBV} ; \mathrm{EPI}=$ echo planar imaging; $\mathrm{FMRI}=$ functional magnetic resonance imaging; $\mathrm{GE}=$ gradient echo; $\mathrm{GM}=$ grey matter; $\mathrm{MION}=$ monocrystalline iron oxide nanocolloid; $\mathrm{NBR}=$ negative $\mathrm{BOLD}$ response; OIS = optical imaging spectroscopy; $\mathrm{PBR}=$ positive $\mathrm{BOLD}$ response; $\mathrm{ROI}=$ region of interest; SAR = specific absorption rate; SNR = signal to noise ratio; SS-SI-VASO = slice selective slab inversion VASO; TE = echo time; $\mathrm{TI}=$ inversion time; $\mathrm{TR}=$ repetition time; $\mathrm{VASO}=$ vascular space occupancy; $\mathrm{WM}=$ white matter.

Key words: vascular space occupancy, SS-SI-VASO, cerebral blood volume, negative BOLD response, 7 Tesla MRI, vascular compartments

\section{Introduction}

Negative blood oxygenation level dependent (BOLD) responses (NBR) have been observed both in animals and humans. NBR can be caused by several independent mechanisms (Kim and Ogawa, 2012) including inhibitory neurogenically driven decreases in cerebral blood flow (CBF) (Shmuel et al., 2002), vasoconstriction in the absence of decreases in neural activity (Shih et al., 2009), or increases in the cerebral metabolic rate of oxygen $\left(\mathrm{CMRO}_{2}\right)$ with no or insufficient $\mathrm{CBF}$ increases (Schridde et al., 2008; Zappe et al., 2008). Under which circumstances the NBR has a vascular or metabolic origin has remained controversial. For this reason, and due to the potential of the negative signal to shed further light on neurovascular coupling, NBR is in the focus of current research (Hutchison et al., 2013; Mullinger et al., 2014; Schäfer et al., 2012; Smith et al., 2004; Tajima et al., 2010; Vafaee and Gjedde, 2004). Early work suggested that the NBR was a result of non-neurally driven hemodynamic mechanisms, such as vascular steal (Harel et al., 2002; Woolsey et al., 1996). However, recent work obtaining electrophysiological recordings simultaneously with BOLD-based functional magnetic resonance imaging (fMRI), in anesthetized macaque monkeys, suggests that decreases in the $\mathrm{CMRO}_{2}$ and neural activity are the major contributors (>60\%) to NBR (Shmuel et al., 2006). Further studies (Kennerley et al., 2012b; Pasley et al., 2007) leave no room for non-neurally driven CBF contributions to NBR, and suggest that neurovascular coupling is conserved for both the positive and negative BOLD responses. There is now a general consensus that the NBR is accompanied by decreases in CBF and $\mathrm{CMRO}_{2}$, as shown with $\mathrm{fMRI}$ in human visual and motor cortex (Pasley et al., 2007; Shmuel et al., 2002; Stefanovic et al., 2004), with fMRI in monkey visual cortex (Shmuel et al., 2006), and with $\mathrm{fMRI}$ and optical imaging spectroscopy (OIS) in rat somatosensory cortex (Boorman et al., 2010; Kennerley et al., 2012b).

More refined layer-dependent studies of NBR in sensory motor cortex of rats and visual cortex of monkeys suggest that the NBR peaks in deeper cortical layers, while the positive gradient echo (GE) BOLD response (PBR) peaks at the cortical surface (Boorman et al., 2010; Goense et al., 2012). The role of cerebral blood volume (CBV) in NBR has remained elusive. Measurements with OIS and monocrystalline iron oxide nanoparticles (MION) reveal vasoconstriction in regions of NBR in rat somatosensory cortex (Boorman et al., 2010; Kennerley et al., 2012b) and cat extrastriate cortex (Harel et al., 2002). Goense et al. (2012), on the other hand, investigated CBV changes in regions of NBR with vascular space occupancy (VASO) (Lu et al., 2003; Yang et al., 2004) and MION in visual cortex of monkeys. They reported a significant and surprising increase 
of CBV in deeper layers of the cortex during inhibitory tasks (Smirnakis et al., 2007). Follow-up studies suggest that this increase of CBV in regions of interest (ROIs) showing NBR is specific to stimulus and area (Bohraus et al., 2013). These and earlier studies (Smirnakis et al., 2007) suggest that BOLD and CBV do not necessarily represent equivalent $\mathrm{FMRI}$ processes and mechanisms.

In contrast to animal research, the role of CBV with regard to PBR and NBR has not yet been investigated in the human brain. In this study, a recently developed multi-echo CBV-sensitive Slice-Saturation Slab-Inversion Vascular Space Occupancy (SS-SI-VASO) variant with BOLD correction (Huber et al., 2013b) was used to investigate the spatiotemporal characteristics of the hemodynamic response during stimuli that elicit positive and negative BOLD signal changes in human brain at 7T. The goal of this study is to understand the underlying hemodynamic mechanisms of both PBR and NBR. This is achieved by breaking down the vascular response from BOLD signal changes, distinguishing larger pial (macro) vessels from microvasculature, and by separately investigating CBV components with arterial and venous-like oxygenation levels.

\section{Materials and Methods}

\subsection{Theory of BOLD-Dependent $T_{2}{ }^{*}$ in SS-SI-VASO}

The signal intensity, $S$, of a parenchyma voxel acquired with blood nulling (bn) and in a control condition without blood nulling (ctr) can be considered as a sum of magnetizations from grey matter (GM) tissue and arterially oxygenated (a) and venously oxygenated (v) blood:

$$
S_{b n / c t r} \sim \sum_{i \in\{G M, a, v\}} V_{i} \rho_{i} M_{i}\left(T I_{b n / c t r}\right) e^{-\frac{T E}{T_{2, i}^{*}}}
$$

where $V_{i}, \rho_{i}$, and $M_{i}$ denote the volume, relative proton density, and the $z$-magnetization of GM and blood within a voxel respectively. $T I$ is the inversion time and $T E$ is the echo time. In SS-SI-VASO, BOLD-contaminated VASO images with blood nulling are acquired interleaved with purely BOLD-weighted control images without blood nulling.

Extravascular BOLD contaminations are assessed and eliminated by dynamic division of images obtained with and without blood nulling. By means of the division, the transverse relaxation term cancels out, and the resulting signal is dependent on $M_{z}$ only (Huber et al., 2013b).

$$
S^{\prime} \approx \frac{M_{G M} e^{-\frac{T E}{T_{2, G M}^{*}}}}{M_{G M} e^{-\frac{T E}{T_{2, G M}^{*}}}+M_{a} e^{-\frac{T E}{T_{2, a}^{*}}}+M_{v} e^{-\frac{T E}{T_{2, G M}^{*}}}} \approx \frac{M_{G M} e^{-\frac{T E}{T_{2, G M}^{*}}}}{\underbrace{\left(M_{\text {par }}\right)}_{\text {const. }} e^{-\frac{T E}{T_{2, p a r}^{*}}}}
$$

However, this formulation does not consider the fact that there are different blood compartments with different baseline $T_{2}{ }^{*}$ values. Thus, we need to extend the model to distinguish blood components with long and short $T_{2}{ }^{*}$ values.

\subsection{1. $\quad T_{2}{ }^{*}$ Model to Estimate 'Arterial' and 'Venous' CBV}

It is well known that there is a direct correlation between intravascular blood $T_{2}{ }^{*}$ and oxygenation level (Ivanov et al., 2013). In the present study, the multi-echo VASO sequence enabled comparisons between the $T_{2}{ }^{*}$ of parenchyma (tissue and vessels) and GM (tissue without vessels), which were used to estimate the $T_{2}{ }^{*}$ of the blood component showing the 
majority of the blood volume changes. This was further evaluated to estimate whether blood volume changes occur in the portion of blood volume with longer baseline $T_{2}{ }^{*}$, which we denote by 'arterial' oxygenation values, or in the portion blood volume with shorter $T_{2}{ }^{*}$, which we denote as 'venous' oxygenation. Since 'arterial' and 'venous' blood portions are here considered based on their oxygenation level and not on anatomical structure, the terms 'arterial' and 'venous' are denoted in quotes. The separate consideration of 'arterial' and 'venous' CBV change based on their $T_{2}{ }^{*}$ can be regarded as distinguishing between BOLDspecific and BOLD-nonspecific CBV changes (Chen and Pike, 2010).

A comprehensive quantitative model of the algorithm to separate the 'arterial' and 'venous' compartment of blood volume change is provided in the appendix. Estimation of venously oxygenated CBV change can be intuitively understood as follows; with the procedure of dynamic division, SS-SI-VASO is sensitive to volume changes of the blood component that is nulled in one condition (bn) and not nulled in the other condition (ctr). Hence, for very short $T E$, both 'arterial' and 'venous' blood compartments are nulled for the bn condition, but in the control condition they both contribute to the signal. Using a longer TE (e.g. TE > $30 \mathrm{~ms}$ ), there is a difference in signal arising from 'arterial' and 'venous' blood. For long TEs, 'arterial' blood will be nulled for the bn condition, but not for the control condition-while 'venous' blood will be nulled in both conditions. In the blood nulled condition, 'venous' CBV is nulled due to $T_{1}$ selective nulling of the VASO contrast. In the control condition 'venous' blood contributions will be highly suppressed due to fast $T_{2}{ }^{*}$ relaxation and dephasing of signal from deoxygenated blood ('venous' $T_{2}{ }^{*} \approx 12 \mathrm{~ms}$ (Ivanov et al., 2013)). When the signal intensities of these two images are divided, 'venous' CBV change does not result in functional VASO signal change. 'Arterial' blood volume change, on the other hand, which is suppressed in the bn condition only, can contribute to the functional contrast.

Hence, SS-SI-VASO reflects the total CBV change at short TE and arterial-weighted CBV changes at longer TE. A comparison of these, almost simultaneously acquired contrasts can be used to estimate 'venous' CBV change.

For quantitative estimates of 'arterial' and 'venous' CBV changes, literature values of 'arterial' and 'venous' $T_{2}{ }^{*}$ were used (appendix). In this study, 'arterial' and 'venous' CBV were defined by their $T_{2}{ }^{*}$ values and oxygenation characteristics, and not by an anatomical vessel classification. Hence, we have assumed that changes in 'venous' CBV take place in microvasculature already containing a significant concentration of deoxyhemoglobin.

\subsection{Image acquisition}

SS-SI-VASO was implemented on a Siemens MAGNETOM 7T scanner (Siemens Healthcare, Erlangen, Germany). For radiofrequency (RF) transmission and reception, a 24-channel receive and circularly polarized single-channel transmit head coil (Nova Medical, Wilmington MA, USA) was used. To circumvent the effects of inflow of fresh (non-inverted) blood magnetization into the microvasculature of the imaging slice, which can be problematic in VASO at 7T (Hua et al., 2013), the inversion efficiency of the inversion pulse was reduced, so that the blood nulling time of the VASO sequence was shorter than the arterial arrival time. In order to achieve proper inversion despite inhomogeneity of the RF field $B_{1}$ and limitations 
imposed by the specific absorption rate (SAR), a TR-FOCl adiabatic inversion pulse (Hurley et al., 2010) was implemented and redesigned to achieve partial inversion in a $B_{1}$-independent manner (Huber et al., 2013b). Data were acquired in five axial slices aligned along the calcarine sulcus with a two-dimensional single-shot multi-gradient echo planar imaging (EPI) readout. Sequence parameters were: $T E_{1} / T E_{2} / T E_{3} / T I_{1} / T I_{2} / T R=12 / 32 / 52 / 1000 / 2500 / 3000$ ms, adjusted inversion efficiency $=85 \%$, partial Fourier factor $=5 / 8$, Grappa factor 3 . Depending on the subjects' brain size, the nominal resolution was between $1.3 \times 1.3 \times 1.5 \mathrm{~mm}^{3}$ and $1.5 \times 1.5 \times 1.5 \mathrm{~mm}^{3}$. Maps of $B_{1}$ and the static field amplitude $B_{0}$ were acquired with a modified actual flip angle imaging (MAFI) (Boulant et al., 2009) method for each subject to ensure proper performance of the inversion pulse used (data not shown).

For the purpose of estimating voxel-wise tissue composition independent of distortions, further inversion recovery measurements with multiple inversion times $\left(T I_{1} S\right)$ of $36 / 200 / 300 / 1200 / 1500$ ms were performed with acquisition parameters otherwise identical to the functional scans. Four additional experiments were conducted to measure CBF changes in ROIs of PBR and NBR. To account for SAR- and $B_{1}$-constraints of pulsed arterial spin labeling (PASL) using a head coil at 7T, a TR-FOCI (Hurley et al., 2010) pulse was implemented in a FAIR-QUIPSSII (Kim, 1995; Wong et al., 1998) sequence. Single-shot double-echo EPI readout was used for image acquisition. Further sequence parameters were: nominal resolution $=3 \times 3 \times 3 \mathrm{~mm}^{3}, T E_{1} / T E_{2} / T I_{1} / T I_{2} / T R=8.2 / 19.4 / 700 / 1700 / 3000 \mathrm{~ms}$. All procedures of this study were approved by the Ethics Committee of the University of Leipzig. Informed written consent was given by all volunteers.

In order to compare VASO results acquired in humans with results from monkeys at higher resolutions, the same SS-SI-VASO sequence used in the human experiments was implemented on a monkey model. These comparisons help in distinguishing between influences of methodology or experimental setup, and can provide additional insights in the interpretation of the human results in the light of previous studies. Hence, SS-SI-VASO, as described above, was implemented on a 7T vertical primate scanner (Bruker Biospec 70/60v, Bruker Biospin $\mathrm{GmbH}$, Ettlingen, Germany) and used in four experiments (imaging the same monkey) employing the experimental setup described in Goense et al. (2010). For further details of experimental hardware (e.g. magnet and RF coils) and animal preparation (e.g. anesthesia and rotating ring stimulus presentation) please refer to (Goense et al., 2012; Logothetis et al., 1999; Pfeuffer et al., 2004). The adapted SS-SI-VASO parameters were $0.6332 \times 0.75 \times 3 \mathrm{~mm}^{3}$ nominal voxel size and $T E / T I_{1} / T_{2} / T R=7.8 / 785 / 2285 / 3000 \mathrm{~ms}$. Experiments were approved by the local authorities (Regierungspräsidium BadenWürttemberg, Germany) and were in full compliance with the guidelines of the European Community (EUVD 86/609/EEC) for the care and use of laboratory animals.

\subsection{Experimental Setup}

Three different $12 \mathrm{~min}$ visual stimulation paradigms (alternating 30 s rest vs. 30 s stimulation) were used to investigate BOLD signal and CBV changes in regions of PBR and NBR in 17 healthy human volunteers ( 8 female, 21 to 32 years old). Two of the paradigms consisted of a full-field flickering checkerboard $(8 \mathrm{~Hz})$ and a small circle flickering checkerboard $(8 \mathrm{~Hz})$. The small flickering checkerboard paradigm (Wade and Rowland, 2010) was chosen in this 
study because it induced stronger NBR in pilot experiments compared to small ring stimulation paradigms (Pasley et al., 2007; Shmuel et al., 2006; Shmuel et al., 2002). The full field stimulation paradigm was used for comparison of the same cortical regions during different stimulation paradigms. For the sake of comparison with recently published results in monkeys, a rotating ring checkerboard (rotation $2 \pi / 3 \mathrm{rad} / \mathrm{s}$, switching rotation direction every 1.5-6 s) was also presented (Goense et al., 2012; Shmuel et al., 2006) (Fig. 1). Based on electrophysiology experiments in monkeys with similar stimuli (Shmuel et al., 2006), we assume that the stimuli used here induce a neural response that is either predominantly excitatory or inhibitory, in regions of PBR and NBR, respectively. However, at the level of individual neurons, both excitation and inhibition are taking place in both NBR and PBR. The terms 'excitatory' and 'inhibitory' are thus used here to describe the stimulation paradigms and their response with regard to their net response within the considered brain regions analogous to earlier studies (Stefanovic et al., 2004).

To control attention and visual fixation, subjects performed a concurrent task in which they responded to subtle color changes of a barely visible fixation point for the duration of the experiments.

\subsection{Data analysis}

The data analysis procedure is graphically summarized in Fig. 1. Magnetic resonance images were motion corrected using SPM8 (Welcome Department, University College London, UK). Statistical analysis was done using FSL Feat (Version 5.98) (Worsley, 2001). In order to estimate voxel-wise tissue composition independent of slice orientation and image distortions, data from multiple $\mathrm{TI}$ experiments were used to generate fractional volume maps of GM, white matter (WM) and cerebrospinal fluid (CSF) (Shin et al., 2010). Assumed $T_{1}$ values were $T_{1, W M} / T_{1, G M} / T_{1, C S F}=1.4 / 1.9 / 4.2 \mathrm{~s}$. Functional ROIs were defined based on the BOLD data as a cluster of voxels having $z$-values above 2.0 and a significance level of $p<$ 0.05 (automatically corrected for multiple comparisons in FSL based upon GRF theory), after smoothing with a kernel size of $1 \mathrm{~mm}$. All further analysis was done in these ROls defined from BOLD data meaning that the same ROls were used for analyzing BOLD and VASO data. Analysis was done on unsmoothed data with in-house written $\mathrm{C}++$ algorithms using libraries of GSL (Galassi and Gough, 2006) and ODIN (Jochimsen and von Mengershausen, 2004).

\subsubsection{Surface Detection}

Variations of GM voxels $T_{1}$ resulting from partial voluming of CSF and WM was used to distinguish voxels containing GM and CSF $\left(T_{1}>T_{1, G M}\right)$ from voxels containing $G M$ and WM $\left(T_{1}<T_{1, G M}\right)$ (Huber et al., 2013b). This separation algorithm is based on the assumption that at the resolution used, simultaneous partial voluming of any voxel with both WM and CSF does not occur.

Because voxels with more CSF partial voluming also have a higher probability of containing superficial cortical layers and pial vessels, these voxels are hereafter referred to as surface GM voxels, in contrast to deeper GM voxels. The threshold of CSF partial voluming to separate the two subsets within the functional ROIs is adjusted such that the numbers of 
surface and deeper GM voxels are equal. This is done to avoid biases across subjects with different curvature.

Considering a nominal voxel size of $1.3-1.5 \mathrm{~mm}$, the voxel size is $70 \%-80 \%$ of the cortical thickness of V1 of $1.8 \pm 0.2 \mathrm{~mm}$ (Jiang et al., 2009). This resolution is thus insufficient to resolve laminar features, but adequate to estimate the influence of surface vasculature. Considering the laminar dependence of total CBV at rest, surface voxels are assumed to have 12\% higher baseline CBV than deeper layers (Kennerley et al., 2005; Zhao et al., 2006) (see also discussion and Fig. 3D). Average baseline CBV within GM was assumed to be $5.5 \%$ (Lu et al., 2013).

\section{Results}

\subsection{Stimulus Setup:}

Using an eye tracker inside the MRI bore, we observed small $\left(1^{\circ}-1.5^{\circ}\right.$ of visual field) saccadic eye movements during each $12 \mathrm{~min}$ experiment. These saccadic movements were slightly smaller than the central circles of the stimulation paradigms. When asked, subjects reported an after-image detectible for 2-10s with the small flickering stimulation paradigm and 20-30s with the rotation ring stimulation paradigm.

\subsection{Spatial Activity Maps}

Significant positive BOLD and corresponding negative VASO signal changes were detected for the rotating ring and small flickering stimulation paradigms in all subjects. For the rotating ring stimulation paradigm, in three of the 17 subjects, significant negative BOLD signal and positive VASO signal changes were detected in one hemisphere only, most probably due to suboptimal positioning of the imaging slices. The regions of interest (ROIs) of activation and deactivation are remarkably similar in maps of BOLD and VASO signal change; the retinotopic organization of visual cortex can clearly be identified in the statistical activation maps (Fig. 2). The GM voxels identified as significantly modulated by the three tasks contained average volume distributions of $19 \% \mathrm{WM}, 68 \% \mathrm{GM}$ and $12 \% \mathrm{CSF}$.

The number of voxels in ROls of NBR was significantly $(p<0.0001)$ smaller for the rotating rings stimulation paradigm (average number of voxels 81 ) compared to the small flickering checkerboard paradigm (average number of voxels 960 ) with resultantly decreased SNR.

\subsection{Surface and Deeper Laminae}

Differences in cortical curvature within ROls of PBR and NBR and differences in hemodynamic response in different cortical areas can result in systematic errors when comparing signal changes across activated and deactivated regions. To avoid such bias, positive and negative responses were considered in the same ROls, but for different stimulation paradigms. Positive (excitatory) and negative (inhibitory) responses refer to fullfield and small flickering checkerboard stimulation, respectively. Positive gradient echo (GE) BOLD signal change was largely dominated by surface GM voxels. CBV on the other hand did not show this pattern and was not significantly different between surface and deeper GM voxels (Fig. 3A). During inhibitory tasks, NBR amplitude was larger $(p<0.05)$ in deeper laminae (Fig. 3B), as observed previously (Boorman et al., 2010; Goense et al., 2012). 
Vasoconstriction during inhibitory tasks is highly dominated by surface GM voxels ( $p<$ 0.001) (CBV change in Fig. 3B).

Regarding the sensitivity to surface vessels and deeper laminae, the change of $C_{B V}$ has a clear signature. The relative 'venous' contribution is significantly $(p<0.001)$ higher in deeper GM voxels without larger vessels (40\%) compared to the surface GM voxels containing such vessels (19\%) (Fig. 3C).

\subsection{Time Course Analysis}

VASO-CBV and BOLD signal time courses during the small flickering checkerboard stimulation paradigm are shown for ROls with PBR and NBR in Figs. $4 A$ and $4 B$, respectively. The time at which the BOLD signal crosses the baseline after stimulus cessation in ROls of NBR $(2.9 \mathrm{~s} \pm 1.7 \mathrm{~s})$ is significantly $(\mathrm{p}<0.001)$ earlier compared to that of PBR ROls $(7.6 \mathrm{~s} \pm$ $2.8 \mathrm{~s}$ )-consistent with previous observations (Goense et al., 2012; Kennerley et al., 2012b; Shmuel et al., 2006). When the mean signal is averaged across each respective ROI, both BOLD signal and CBV changes have the same sign in ROls with NBR as compared to ROls with PBR. The mean amplitude of both CBV change and BOLD response in ROls of NBR is (35 \pm 5 )\% of the amplitude of the ROIs of PBR, suggesting a conserved neurovascular coupling for both cases.

\section{5. 'Arterial' and 'Venous' CBV}

To avoid the bias of potentially different vascularization in different parts of the visual cortex (Weber et al., 2008), 'arterial' and 'venous' blood volume changes $\left(\Delta C B V_{a / v}\right)$ are considered in one part of the cortex only, namely in ROls that show NBR in response to the small circle flickering checkerboard visual stimulation. These GM voxels are considered during excitatory tasks (full-field stimulation) and inhibitory tasks (small circle stimulation). During the excitatory task, the total CBV change, $\triangle \mathrm{CBV}_{\text {tot }}$, of $(23.7 \pm 2.2) \%$ is composed of (16.6 \pm 1.6$) \%$ 'arterial' $\triangle \mathrm{CBV}_{\mathrm{a}}$ and $(7.1 \pm 2.4) \%$ 'venous' $\triangle \mathrm{CBV}_{\mathrm{v}}$. Therefore, the relative composition of $\triangle \mathrm{CBV}_{\text {tot }}$ is $71 \%$ 'arterial' $\triangle \mathrm{CBV}_{\mathrm{a}}$ and $29 \%$ 'venous' $\triangle \mathrm{CBV}_{\mathrm{v}}$. 'Arterial' $\mathrm{CBV}_{\mathrm{a}}$ has a faster response and a faster post-stimulus baseline crossing, compared to the 'venous' $\mathrm{CBV}_{\mathrm{v}}$ response (Fig. 5B), as observed previously in animals (Kennerley et al., 2012a; Kim and Kim, 2011; Zong et al., 2012). 'Venous' $\triangle C B V_{v}$ contribution appears negligibly small compared to $\triangle C B V_{\text {tot }}$ (Fig. $5 \mathrm{C}$ ) during the inhibitory task, as $\triangle \mathrm{CBV}_{\mathrm{v}}$ is not significantly different from zero.

\section{6. $\mathrm{CBF}$}

ASL-FAIR experiments provide a sufficiently high signal-to-noise-ratio (SNR) to estimate CBF changes in ROls of PBR and NBR at 7T (Fig. 6B). CBF time courses show similar dynamics compared to BOLD or 'arterial' $\mathrm{CBV}_{\mathrm{a}}$. There is a significant CBF increase in ROIs with PBR and a significant CBF decrease in ROls with NBR, as observed earlier in humans (Pasley et al., 2007; Shmuel et al., 2002; Stefanovic et al., 2004).

\subsection{Rotating Ring Stimulation:}

The time courses in Fig. 7 refer to the ROls with NBR during the rotating ring stimulation paradigm. Activation and deactivation refer to full-field flickering checkerboard and ring stimulation within these ROIs. Despite the smaller functional contrast-to-noise ratio (CNR) 
in the ring paradigm, the same temporal features of positive and negative responses can be seen, i.e. faster post-stimulus baseline crossing as well as the laminar-dependent characteristics in CBV and BOLD signal changes. Aside from the different amplitudes of the time courses, there is not significant difference between surface and deeper GM voxels.

\subsection{Monkey Experiments}

Positive and negative BOLD responses during a rotating ring stimulation paradigm could be detected with SS-SI-VASO in monkey brain (Fig. 8). In the depicted sagittal slice, NBR in areas correspond to the ring of grey background, and PBR regions correspond to the outer checkerboard ring. Maps of BOLD corrected VASO signal change indicate increase in CBV in regions of NBR, consistent with earlier experiments in monkeys but in contrast to the human results in this study.

\section{Discussion}

The data presented in this study suggest that the effects of specific vasculature compartments on the BOLD signal changes can be investigated during excitatory and inhibitory tasks non-invasively in humans at 7T. With the relatively high SNR of the SS-SIVASO method used here, the spatiotemporal characteristics of the interplay between BOLD and vasculature could be identified, such as differences in time courses and signal origin from different vascular compartments. Even though the voxel volume in FAIR experiments ( 3 $\times 3 \times 3 \mathrm{~mm}^{3}$ ) was eight times larger than that used with SS-SI-VASO (d $\left.1.5 \times 1.5 \times 1.5 \mathrm{~mm}^{3}\right)$, the CNR of the functional CBV was still larger than the CNR of the functional CBF. This suggests that SS-SI-VASO can be a useful tool in obtaining high SNR non-BOLD hemodynamic responses at high fields, especially when no separate labeling coil for arterial spin labeling is available.

Given that the voxel size in this study was comparable to the cortical thickness (approx. $72 \%$ ), surface GM voxels are likely to contain larger superficial arteries and veins as well as microvasculature of the middle and upper layers. Deeper GM voxels will contain microvasculature of the cortical tissue without influence from larger superficial vessels (Duvernoy et al., 1981; Weber et al., 2008). The additional separation into 'arterial' and 'venous' CBV provides indications which type of vessels are responsible for the detected signal changes, arteries or veins.

The larger positive BOLD response at the surface (Fig. $3 \mathrm{~A}$ ) results most probably from the typical accumulation of blood oxygenation changes at the cortical surface, where the 'venous' blood leaves the cortex, and from the well-established GE BOLD sensitivity to larger veins (Kim and Ogawa, 2012). The fact that VASO contrast is independent of whether the GM voxels contain larger surface vessels (Fig. $3 \mathrm{~A}$ ) suggests that its contrast has higher specificity to the microvasculature, similar to other CBV-sensitive modalities (Jin and Kim, $2008 \mathrm{a}, \mathrm{b})$. The same was also seen in high-resolution $\left(500 \times 500 \mu \mathrm{m}^{2}\right)$ data obtained in macaques in the current study (data not shown). Figure $3 \mathrm{C}$ shows a relatively larger proportion of 'arterial' CBV change in surface GM voxels compared to deeper GM voxels, while the 'venous' contribution to the total CBV change occurs predominantly in deeper tissue voxels. This is consistent with the fact that the contribution of larger veins at the 
surface is small compared to the overall CBV change, dominated by arterioles and capillaries (Hillman et al., 2007; Lee et al., 2001). The larger arterioles close to the cortical surface, on the other hand, can contribute to the CBV change, in addition to the smaller arterioles and capillaries.

The interpretation of the results for inhibitory tasks (Fig. 3B and 5D) is less obvious. Negative BOLD contrast shows smaller signal changes in surface voxels containing large superficial veins, and vasoconstriction is greatest in larger superficial arteries. The loss of surface dominance in the negative BOLD response can partly arise from contamination of pial vessels draining adjacent excited regions and passing though surface voxels of deactivated regions. This effect can explain artifactual BOLD signal changes up to about $3 \mathrm{~mm}$ away from the activated region (Turner, 2002). Independently, it might also result from different underlying layer-dependent inhibition compared to excitation. The different laminar dependence might be also associated with an altered interplay of responses in different compartments of the vascular tree.

There are several indications in the CBV data that suggest altered dynamics within the different vascular compartments during inhibitory tasks as compared to excitatory tasks. Firstly, during inhibitory tasks the CBV change is dominant in surface GM voxels (Figs. 3B and 7). This is in contrast to the excitatory case, where the CBV change is similar within surface and deeper GM voxels. Secondly, data shown in Figs. 5C and 5D suggest that the corresponding CBV decrease comes only from fully oxygenated arterial vessels, and not from the less oxygenated microvasculature and venules. Lastly, the faster CBV return to baseline after inhibitory stimulation is a further indication that the CBV decrease for the inhibitory task has an arterial origin.

If the neurovascular coupling were the same during excitatory and inhibitory tasks, it leads one to expect the same hemodynamic control mechanisms and the same interplay between the different vascular compartments. However, the different spatiotemporal hemodynamic response and different interaction of vascular compartments suggest differing neurovascular coupling during excitatory and inhibitory tasks. This might be associated with hemodynamic control mechanisms discussed earlier (Bandettini, 2012; Goense et al., 2012; Shmuel et al., 2006).

It must be noted that VASO is a methodology only proportional to CBV change. In order to estimate the relative CBV change from the VASO signal change, a value of $\mathrm{CBV}_{\text {rest }}$ needs to be assumed (usually 5.5\%) (Lu et al., 2013). Although microvascular $\mathrm{CBV}_{\text {rest }}$ is relatively homogeneously distributed across layers (Kim et al., 2013; Weber et al., 2008), when macrovasculature is also included the total $\mathrm{CBV}_{\text {rest }}$ was found to be largest at the cortical surface, decreasing with cortical depth (Kennerley et al., 2005; Zhao et al., 2006). Higher CBV $_{\text {rest }}$ at the cortical surface is associated with a smaller relative $\triangle C B V$ for the same VASO signal change. Figure $3 \mathrm{D}$ depicts the reduction in the calculated $\triangle \mathrm{CBV}$ in the surface voxels when the high macro-vasculature $\mathrm{CBV}_{\text {rest }}$ in surface layers taken into account.

\subsection{Limitations in Separation of 'Arterial' and 'Venous' CBV Change}


Given the complexity of the vascular architecture, it is necessary to discuss the implications of the non-linear arterio-venous separation model.

Aside from the assumed $T_{2}{ }^{*}$ values, the model does not rely on assumptions regarding the vascular dynamics, interaction of vascular compartments, or spatial distribution of the vasculature within the MR images. Hence, it is expected to work across voxels with various vascular architectures and contributions of vascular compartments.

For every activation state (time point) four images are required (double echo images without blood nulling and double echo images with selective blood nulling) to calculate the 'arterial' and 'venous' CBV change. Hence, the method requires a relatively high temporal resolution of the BOLD and VASO signals. Linear interpolation between the consecutive image acquisitions and the short interval of $1.5 \mathrm{~s}\left(\mathrm{TI}_{2}-\mathrm{TI}_{1}\right)$ used in this study enables the estimation of 'arterial' and 'venous' CBV time courses even during the transition periods between rest and activation.

'Arterial' and 'venous' blood are defined here based on their relaxation times and thus on their oxygenation level, and not by their anatomical architecture. Therefore, oxygenationlevel defined compartments do not necessarily coincide with anatomically defined compartments. Oxygen saturation is highest in large arteries, decreasing downstream to arterioles and capillaries (Yaseen et al., 2011). Within the venules and veins, the oxygen saturation is nearly constant, independent of vessel size (Yaseen et al., 2011). In fact, the largest drop in oxygen saturation appears to occur within the arterioles. Therefore, changes in 'venous' (65\%-oxygenated) CBV, estimated in the proposed model, might arise to a small part from these arterioles as well as from capillaries, venules and veins. In arteries and arterioles during stimulation, the majority of the total CBV change occurs almost entirely due to an increase in the amount of fully oxygenated blood (Kennerley et al., 2012a). The CBV changes in post-arterial compartments, however, are likely to mostly contain 'venous' CBV. Since the model is based on $T_{2}{ }^{*}$ of two predefined oxygenation levels of arteries $(Y=$ $98.4 \pm 0.7 \%$ ) and veins ( $Y=64 \pm 6 \%$ ) (Lu et al., 2008; Wehrli et al., 2014), any oxygenation level in between these values is treated from the model as a superposition of a fraction of fully oxygenated arterial blood and a fraction of $64 \%$ oxygenated 'venous' blood.

Intravascular changes of $T_{2}{ }^{*}$ can alter the sensitivity of the separation method of 'arterial' and 'venous' CBV. During a 5\% hypercapnia challenge, the 'venous' oxygenation changes from $(64 \pm 6) \%$ to $(72 \pm 6) \%$ with a corresponding $T_{2}{ }^{*}$ change of $4 \mathrm{~ms}$ (intravascular BOLD effect) (Ivanov et al., 2013). The variations in $T_{2}{ }^{*}$ during stimulation are expected to be of the same order of magnitude (Huber et al., 2013a). This intravascular BOLD contamination suggests that between activation and rest, the method of separation of 'arterial' and 'venous' changes its sensitivity in each compartment. Since the stimulus-induced $T_{2}{ }^{*}$ change of $4 \mathrm{~ms}$ is much smaller than the $T_{2}{ }^{*}$ difference between 'arterial' and 'venous' CBV (37 ms and $12 \mathrm{~ms}$ (Ivanov et al., 2013)), this contamination is believed to be negligible. In the appendix, this contamination is estimated and corrected for, using the variable $C_{1}$.

Uncertainties in the literature values of arterial and venous oxygen saturation can affect the 'arterio'-'venous' separation model. Arterial and venous oxygen saturation variations are (98.4 \pm 0.7$) \%$ and $(64 \pm 6) \%$ (mean \pm standard deviation across subjects) (Lu et al., 2008; Wehrli et al., 2014) with corresponding uncertainties in $T_{2}{ }^{*}$ of $\pm 2 \mathrm{~ms}$ (Ivanov et al., 2013). These variations come from subject dependent oxygen partial pressures (Gray and Steadman, 1964). The corresponding uncertainty of 'arterial' and 'venous' CBV in individual 
subjects, estimated at about $15 \%$, is smaller than the inherent noise level of the estimation of arterial and venous $C B V$ changes of $34 \%$ (see uncertainty of $\triangle C B V_{v}$ of $7.1 \pm 2.4 \%$ in section 3.5). The inter-subject variation of venous baseline oxygen saturation values would thus result in an additional uncertainty of $\triangle \mathrm{CBV}_{\mathrm{v}}$ estimations of $1.0 \%$, that is, $\triangle \mathrm{CBV}_{\mathrm{v}}=7.1 \pm 2.4$ (data noise) \pm 1.0 (biological oxygenation variations) \%. In this study the analysis was performed by averaging across subjects, thus neglecting individual variations in venous oxygenation level.

The sensitivity of the separation method is significantly smaller than the sensitivity of VASO. The estimation of 'arterial' and 'venous' CBV is based on the VASO signal changes as a function of TE (Fig. 5A). Considering the small size of it and comparing it to the size of absolute VASO signal change, it suggests that the sensitivity of the separation method is about one twelfth of the sensitivity of VASO contrast and one twentieth of the sensitivity of BOLD contrast.

Estimation of 'arterial' and 'venous' CBV changes has not been done on a single subject basis but across all subjects, which has several implications regarding its limitations. (A) Intersubject variation is not considered. (B) The interplay between 'arterial' and 'venous' oxygenation and blood volume can only be investigated in predefined ROls as additional to other imaging contrasts, and not as an independent method. (C) Due to the nonlinearity of the model, it has no distributive property (average $\left(\operatorname{model}\left(x_{i}\right)\right) \neq \operatorname{model}\left(\operatorname{average}\left(x_{i}\right)\right)$ ), therefore the result is dependent on the point of averaging in the evaluation chain. Due to the nonlinear signal transformation of the model, the nonlinear transformation of the substantial noise level and the corresponding noise amplification in individual subject data could render subject-wise analysis impossible. However, since the changes in $T_{2}{ }^{*}$ are small compared to their absolute values (see also appendix), the results of this study can be considered to be in the linear regime of the non-linear model. Hence, prior averaging with subsequent application of the model seems appropriate.

\subsection{Comparison with Earlier Studies}

\subsubsection{Arterial and Venous Blood Volume Dynamics}

There are a number of studies investigating the relationship between arterial and venous vasculature measured with various MR and non-MR modalities. In most of these studies, as listed below, there is consensus that the arterial contribution to the CBV change is much larger than the venous CBV contribution. For example, relative venous contributions of $34 \%$ were measured with 2D OIS in rat somatosensory cortex (Berwick et al., 2005), 30\% with perfluorocarbon MRS during hypercapnic perturbation (Lee et al., 2001), and 0-12\% with two-photon microscopy in mouse somatosensory cortex (Hillman et al., 2007; Takano et al., 2006). Comparing iron-based MR contrast agent and $C B V_{a}$-sensitive MRI methods (Kim and Kim, 2005), the arterial CBV change was shown to account almost alone for the total CBV change (Kim et al., 2007) with increasing venous contribution only for long stimulus durations (Kim and Kim, 2011). Non-invasive human studies, using oxygen as an MR contrast agent, have shown that venous CBV can apparently even decrease in activated brain regions (Blockley et al., 2012). This contradicts comparisons of CBV-VASO and CBV $_{\mathrm{v}}-\mathrm{VERVE}$ (Stefanovic and Pike, 2005) showing a $\mathrm{CBV}_{\mathrm{v}}$ contribution of $25 \%$ (Cohalan et al., 2009). In summary, the relative 'venous' contribution of $29 \%$ measured in this study lies within the previously reported range of values given in the literature. 
With respect to the temporal dynamics of the CBV response, delayed recovery of 'venous' $\mathrm{CBV}_{\mathrm{v}}$ is often proposed to explain the delayed return to baseline after stimulus cessation (Buxton, 2010; Kim and Kim, 2011), in agreement with the balloon or Windkessel model (Buxton et al., 1998; Mandeville et al., 1998). The slow return to baseline of 'venous' CBV (Fig. 5B) in the present study, while 'arterial' $\mathrm{CBV}$ and CBF time courses show faster returns to baseline (Figs. $5 B$ and $6 C$ ) is consistent with venous balloon effects. However, the two human studies investigating $\mathrm{CBV}_{\mathrm{v}}$ dynamics failed to report a delayed venous return to baseline (Blockley et al., 2012; Stefanovic and Pike, 2005). The significantly slower poststimulus return to baseline of 'venous' CBV shown in this study has been seen only in animal studies so far (Kim and Kim, 2011; Mandeville et al., 1998).

\subsubsection{CBV change in ROIs of NBR}

The occurrence of vasoconstriction with negative BOLD response is consistent with the majority of animal studies (Boorman et al., 2010; Harel et al., 2002; Kennerley et al., 2012b). The fast temporal CBV response shown in Figs. $4 \mathrm{~A}$ and 7 in ROls with NBR is consistent with findings in (Boorman et al., 2010; Kennerley et al., 2012b). By contrast, Harel et al. (2002) report a significantly slower CBV response in ROIs of NBR than in ROIs of PBR.

Since the vascular response underlying NBR can vary across various brain regions (Bohraus et al., 2013) and stimulation paradigms, it could be misleading to directly compare the neurovascular results of this study with studies investigating other cortical regions, for example, extrastriate (Harel et al., 2002), or somatosensory cortex (Boorman et al., 2010; Liu et al., 2011; Pasley et al., 2007; Stefanovic et al., 2004). The most comparable stimulation paradigm was used by Goense et al. (2012). Their results suggest a CBV increase in ROls of NBR. The results of the present study on humans, on the other hand, suggest a CBV decrease in these regions. The differences could be due to: (A) Differences in imaging modalities such as BOLD contamination or inflow effects in VASO could explain the conflicting results; (B) The discrepancy could be a result of differences in the experimental setup.

Comparison of the SS-SI-VASO in humans and monkeys allows distinction between these possibilities. A representative map of BOLD- and VASO signal changes in a monkey scan is shown in Fig. 8. Throughout all four SS-SI-VASO scans in the monkey, negative VASO signal changes were seen in ROls of NBR, indicating a CBV increase in these regions. This is consistent with results presented by Goense et al. (2012), but it contradicts the results in humans presented here. Since the same MR sequence is used in both experiments, we hypothesize that the observed differences result from differences in the experimental setup, such as poorer visual fixation in humans, differences due to the additional task-related feedback in humans (since they are awake), or a smaller extent of negative ROls (species differences), differences in arterial blood pressure due to anesthesia or due to differences in posture (Payne, 2006; Raz et al., 2005), differences of attention (Moradi et al., 2012), or differences in interstitial space and CSF dynamics during anesthesia (Xie et al., 2013) etc. Since the architecture of the cortical (micro) vasculature of striate cortex is very similar in monkeys and humans (Keller et al., 2011; Weber et al., 2008), different vascularization is not 
expected to be responsible for the afore-mentioned differences observed in humans and monkeys.

\subsubsection{Hypothetical Mechanism Underlying Negative BOLD Contrast}

The spatially extended CBV increase in regions of monkey $V 1$ that show negative BOLD responses (Goense et al., 2012) has been discussed in the context of increased venous afterload and increased venous back pressure (Bandettini, 2012; Goense et al., 2012; Smirnakis et al., 2007). When the drainage of blood from ROIs of NBR is hampered due to increased venous back pressure arising from surrounding excited regions, the passively controlled vascular compartments can dilate for hydrodynamic reasons, independently of $\mathrm{CBF}$ decrease and vasoconstriction in the arterial compartments. This hypothetical mechanism provides explanations for several features of the results presented. (A) Venous back pressure can be understood as a local phenomenon. Since in monkeys the negative BOLD ROls have the same spatial scale as the cortical thickness (3-6 mm, see Fig. 8) the venous back pressure could have a stronger effect as compared with humans, where the negative BOLD ROls are much larger in extent (10-30 mm, see Fig. 2 ) and neighboring excited regions are much further away. (B) The venous back pressure can only influence the passive downstream vascular compartments and not the actively controlled arterioles close to the cortical surface. This is consistent with the observation that in ROIs of NBR vasoconstriction is confined to fully oxygenated arteries (Figs. 5C and 5D) close to cortical surface (Figs. 3B and 7) and not in the more passively controlled microvasculature in the tissue.

Comparative fMRI studies between humans and monkeys, for example, using hemi field visual stimulation or varying the size of the negative ROI, could help to investigate the validity and extent of the above hypothesis.

An additional hypothetical mechanism can be associated with the different amplitude of fMRI response in human and monkey experiments. BOLD fMRI responses and neural activity is smaller during anesthesia then in the alert condition (Goense and Logothetis, 2008; Martin et al., 2006). Dependent on the extent of the stimulus (e.g. stimulation frequency), the interplay of inhibitory and excitatory circuits could vary, resulting in positive and negative fMRI response respectively (Logothetis et al., 2010). Hence, the different amounts of cortical input in the different conditions considered here may also cause very different hemodynamic responses.

\section{Conclusions}

We developed methods and models to investigate simultaneously the spatiotemporal characteristics of BOLD contrast and vascular responses during excitatory and inhibitory stimulation in humans. The observed responses were considered with regard to their sources in surface vessels versus microvasculature, and vascular compartments of arterially and venously oxygenated blood. By these means, features of the neurovascular coupling accessible so far only in animal models could be investigated in humans. The observed excitatory and inhibitory characteristics of CBV and BOLD contrast, together with their dependence on cortical depth and 'arterial' vs. 'venous' compartments, are in good agreement with the animal literature. Even though the interplay of mean CBV, CBF and BOLD signal changes is very similar for neural excitatory and inhibitory stimulation, some aspects of the neurovascular coupling are significantly different for the two cases, such as the temporal response, cortical depth 
dependence, and the weighting between 'arterial' and 'venous' contributions. Excitatory stimulation is associated with increased CBV responses in all vascular compartments, while inhibitory stimulation evokes decreases in the hemodynamic response predominantly in the larger superficial arterial vessels. These variations in the relationship between 'arterial' and 'venous' CBV and BOLD changes suggest different underlying hemodynamic control mechanisms for positive and negative BOLD responses.

6. Acknowledgments: We acknowledge helpful discussions with Prof. Dr. Seong-Gi Kim regarding the possible volume change of CSF bordering surface layers. We thank Domenica Wilfling and Elisabeth Wladimirow for helping take care of the volunteers. We are grateful to Daniel Zaldivar, Thomas Steudel, and Deniz Ipek for assistance with the monkey experiments and to Prof. Nikos Logothetis for allowing us to perform the experiments in his laboratory. We thank Christine Tardif for helpful discussions regarding retinotopy. We thank Heike Schmidt-Duderstedt for design of Fig. 1. We thank Enrico Reimer for IT-support regarding MRI data conversion. We are thankful to Benedikt Poser for helping with the implementation of additional echoes into the sequence code. We thank Alexander Kranz for access to a Bruker Paravision 5.1 System needed for validation of the SS-SI-VASO method. The research was supported by the Max Planck Society.

\section{Appendix: Estimation of $\triangle \mathrm{CBV}_{\mathrm{v}}$ in SS-SI-VASO}

As given in Section 2.1, the SS-SI-VASO signal incorporating 'arterial' and 'venous' CBV can be expressed as:

$$
S=\frac{M_{G M} e^{-\frac{T E}{T_{2, G M}^{*}}}}{M_{G M} e^{-\frac{T E}{T_{2, G M}^{*}}}+M_{a} e^{-\frac{T E}{T_{2, a}^{*}}}+M_{v} e^{-\frac{T E}{T_{2, G M}^{*}}}}=\frac{1}{1+\frac{M_{a}}{M_{G M}} e^{-T E\left(R_{2, a}^{*}-R_{2, G M}^{*}\right)}+\frac{M_{v}}{M_{G M}} e^{-T E\left(R_{2, v}^{*}-R_{2, G M}^{*}\right)}}
$$

For simplicity, all magnetizations refer to $z$ magnetizations and are written in units of $M_{\text {tissue; }}$; therefore $M_{G M}=1-C B V$. It can be assumed that the proton densities of tissue and blood are very similar (0.89 vs. $0.87 \mathrm{ml}$ water / $\mathrm{ml}$ tissue (Donahue et al., 2009)). Only zeroth and first order terms are considered on all Taylor expansions. Eq. A1 can be simplified to

$$
S=\frac{M_{a}}{M_{G M}} e^{-T E \Delta R_{a}^{*}}+\frac{M_{v}}{M_{G M}} e^{-T E \Delta R_{v}^{*}} .
$$

Hence,

$$
\begin{aligned}
\Delta S=S^{a c t}- & S^{r e s t}= \\
& =\frac{M_{a}^{a c t}}{M_{G M}^{a c t}} e^{-T E \Delta R_{a}^{*, a c t}}+\frac{M_{v}^{a c t}}{M_{G M}^{a c t}} e^{-T E \Delta R_{v}^{*, a c t}}-\frac{M_{a}^{r e s t}}{M_{G M}^{r e s t}} e^{-T E \Delta R_{a}^{*}, \text { rest }} \\
& -\frac{M_{v}^{r e s t}}{M_{G M}^{r e s t}} e^{-T E \Delta R_{v}^{*, r e s t}}
\end{aligned}
$$

with $R_{a / v}^{*}=\frac{1}{T_{2, a / v}^{*}}$, and $\Delta R_{a / v}^{*, a c t / r e s t}=\frac{1}{T_{2, a / v}^{*, a c t / r e s t}}-\frac{1}{T_{2, G M}^{*, a c t / r e s t} \text {. }}$.

Even in veins during hypercapnia, the change in $T_{2}{ }^{*}$ between activation and rest ( $12-16 \mathrm{~ms}$ ) is much smaller compared to $T_{2}{ }^{*}$ differences between 'arterial' and 'venous' blood (12 - $37 \mathrm{~ms}$ ) (Ivanov et al., 2013). Therefore, $e^{-T E\left(\Delta R_{v}^{*, a c t}-\Delta R_{v}^{*} \text {,rest }\right)} \approx 1-T E\left(\Delta R_{v}^{*, a c t}-\Delta R_{v}^{*, \text { rest }}\right)$. Since 'arterial' CBV is defined here by its $T_{2}{ }^{*}$ value to be fully oxygenated, there is no BOLD effect in arteries: 
$\left(\left(\Delta R_{a}^{*, r e s t}-\Delta R_{a}^{*, a c t}\right)=0\right)$ and Eq. A2 can be simplified to:

$$
\begin{aligned}
& \Delta S= \\
& {\left[\frac{M_{a}^{a c t}}{M_{G M}^{a c t}}-\frac{M_{a}^{r e s t}}{M_{G M}^{r e s t}}\right] e^{-T E \Delta R_{a}^{*}}+\left[\frac{M_{v}^{a c t}}{M_{G M}^{\text {act }}}-\frac{M_{v}^{\text {rest }}}{M_{G M}^{\text {rest }}}\right] e^{-T E \Delta R_{v}^{*}}+\underbrace{\frac{M_{v}^{\text {rest }}}{M_{G M}^{\text {rest }}} T E\left(\Delta R_{v}^{*, a c t}-\Delta R_{v}^{*, r e s t}\right)}_{C_{1}}}
\end{aligned}
$$

$C_{1}$ can be considered as a higher order term that comprises the intravascular BOLD effect. According to the definition of $\Delta R_{a / v}^{*, a c t / r e s t}$ in Eq. A2, it can be rewritten as:

$C_{1}=\frac{M_{v}^{r e s t}}{M_{G M}^{r e s t}} T E\left[R_{v}^{*, a c t}-R_{v}^{*, r e s t}-\left(R_{G M}^{*, a c t}-R_{G M}^{*, \text { rest }}\right)\right]$.

Eq. A3 can be used to explain the features of Fig. 5A. Since 'arterial' blood has a longer $T_{2}{ }^{*}$ than GM, the first term of Eq. A3 is amplified for longer TEs and the 'arterial' CBV change is overestimated. Since 'venous' blood has a significantly shorter $T_{2}{ }^{*}$ than $G M$, the second term of Eq. A3 is suppressed at longer TEs and the 'venous' CBV change is underestimated. Using literature values for 'arterial', 'venous' and $\mathrm{GM} \mathrm{T}_{2}{ }^{*}$, the TE-dependence in time courses of Fig. $5 \mathrm{~A}$ can be used to estimate the 'arterial' and 'venous' contributions to blood volume change according to:

$$
\begin{aligned}
& \Delta S(T E=0)-\Delta S\left(T E^{\prime}\right)= \\
& =\left[\frac{M_{a}^{a c t}}{M_{G M}^{a c t}}-\frac{M_{a}^{r e s t}}{M_{G M}^{r e s t}}\right]+\left[\frac{M_{v}^{a c t}}{M_{G M}^{a c t}}-\frac{M_{v}^{\text {rest }}}{M_{G M}^{\text {rest }}}\right]-\left\{\left[\frac{M_{a}^{a c t}}{M_{G M}^{a c t}}-\frac{M_{a}^{\text {rest }}}{M_{G M}^{\text {rest }}}\right] e^{-T E^{\prime} \Delta R_{a}^{*}}+\left[\frac{M_{v}^{a c t}}{M_{G M}^{a c t}}-\frac{M_{v}^{\text {rest }}}{M_{G M}^{\text {rest }}}\right] e^{-T E^{\prime} \Delta R_{v}^{*}}\right\}= \\
& \frac{C B V_{a}^{a c t}+C B V_{v}^{a c t}}{\left(1-C B V^{a c t}\right)}-\frac{C B V_{a}^{r e s t}+C B V_{v}^{r e s t}}{\left(1-C B V^{r e s t}\right)}- \\
& \left\{\frac{C B V_{a}^{a c t}}{\left(1-C B V^{a c t}\right)} e^{-T E^{\prime} \Delta R_{a}^{*}}-\frac{C B V_{a}^{\text {rest }}}{\left(1-C B V^{\text {rest }}\right)} e^{-T E^{\prime} \Delta R_{a}^{*}}+\frac{C B V_{v}^{a c t}}{\left(1-C B V^{a c t}\right)} e^{-T E^{\prime} \Delta R_{v}^{*}}-\frac{C B V_{v}^{\text {rest }}}{\left(1-C B V^{\text {rest }}\right)} e^{-T E^{\prime} \Delta R_{v}^{*}}\right\}-
\end{aligned}
$$

$C_{1}$

Assuming that the relative $\mathrm{GM}$ volume is small compared to the relative $\mathrm{CBV}(1-C B V \approx 1)$ and considering that any CBV change is either 'arterial' or 'venous' $\left(\triangle C B V_{a}+\triangle C B V_{v}=\triangle C B V\right)$, Eq. A4 can be further simplified to:

$$
\begin{aligned}
& \Delta S(T E=0)-\Delta S\left(T E^{\prime}\right)=\Delta C B V-\left\{\Delta C B V e^{-T E^{\prime} \Delta R_{a}^{*}}+\Delta C B V_{v}\left[e^{-T E^{\prime} \Delta R_{v}^{*}}-e^{-T E^{\prime \Delta R_{a}^{*}}}\right]\right\}- \\
& C_{1}=\Delta C B V\left[1-e^{-T E^{\prime \Delta R_{a}^{*}}}\right]+\Delta C B V_{v}\left[e^{-T E^{\prime} \Delta R_{v}^{*}}-e^{-T E^{\prime \Delta R_{a}^{*}}}\right]-C_{1}
\end{aligned}
$$

Solved for $\triangle \mathrm{CBV}_{\mathrm{v}}$, Eq. A5 becomes:

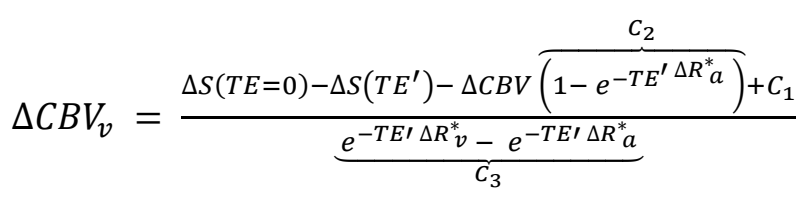

\begin{tabular}{|c|c|c|c|c|c|c|c|c|c|}
\hline$T_{2, a}^{*}$ & $R_{2, a}^{*}$ & $T_{2, v}^{*}$ & $R_{2, v}^{*}$ & $T_{2, G M}^{*}$ & $R_{2, G M}^{*}$ & $\Delta R_{a}^{*}$ & $\Delta R_{v}^{*}$ & $C_{2}$ & $C_{3}$ \\
\hline
\end{tabular}




\begin{tabular}{|c|c|c|c|c|c|c|c|c|c|}
\hline \multicolumn{2}{|c|}{ (Ivanov et al., 2013) } & \multicolumn{2}{|c|}{ (Ivanov et al., 2013) } & \multicolumn{2}{|c|}{ (Donahue et al., 2011) } & \multicolumn{2}{c|}{ Eq. A2 } & \multicolumn{2}{c|}{ Eq. A6 } \\
\hline $37.5 \mathrm{~ms}$ & $26.6 \mathrm{~s}^{-1}$ & $12.2 \mathrm{~ms}$ & $81.7 \mathrm{~s}^{-1}$ & $25.1 \mathrm{~ms}$ & $39.8 \mathrm{~s}^{-1}$ & $-13.1 \mathrm{~s}^{-1}$ & $42.1 \mathrm{~s}^{-1}$ & -0.17 & -0.57 \\
\hline
\end{tabular}

Table A1: Literature relaxation values of single components for separation of 'arterial' and 'venous' CBV change.

By means of Eq. A6 and the literature values given in Table A1, the multi-exponential VASO data can be used to separate 'venous' and 'arterial' CBV changes. According to Eq. A6, signal changes at two different TEs are needed to calculate the 'venous' CBV change. Due to SNR limitations, signal changes with TEs of $12 \mathrm{~ms}$ and $32 \mathrm{~ms}$ were used in this study, neglecting signal changes measured with $T E=52 \mathrm{~ms}$ (Fig. 5A).

\begin{tabular}{|c|c|c|c|}
\hline$C B V_{v}$ & $R_{2, v}^{*, a c t}-R_{2, v}^{*, \text { rest }}$ & $R_{2, G M}^{*, a c t}-R_{2, G M}^{*, \text { rest }}$ & $C_{1}$ \\
\hline (Kim and Ogawa, 2012) & (Ivanov et al., 2013) & (Donahue et al., 2011) & Eq. A3 \\
\hline $70 \%$ of 5.5\% $\left(C B V_{t}\right)=3.85 \%$ & $-22.4 \mathrm{~s}^{-1}$ & $-0.77 \mathrm{~s}^{-1}$ & -0.0098 \\
\hline
\end{tabular}

Table A2: Literature values for estimation of the intra-vascular BOLD term $\mathrm{C}_{1}$.

$C_{1}$ is one order of magnitude smaller than $C_{2}$ and $C_{3}$, which is consistent with the fact that at 7T intravascular BOLD is negligibly small (10\% of the total BOLD signal (Donahue et al., 2011; Martindale et al., 2008)). Here, $C_{1}$ is used as a linear parameter. Neglecting it can lead to an underestimation of the 'venous' CBV change by approx. $16 \%$ (resulting in $\triangle C B V_{v}=6.1 \%$ instead of the true value of $\left.\triangle C B V_{v}=7.08 \%\right)$.

\section{References:}

Bandettini, P.A., 2012. The BOLD plot thickens: sign- and layer-dependent hemodynamic changes with activation. Neuron 76, 468-469.

Berwick, J., Johnston, D., Jones, M., Martindale, J., Redgrave, P., McLoughlin, N., Schiessl, I., Mayhew, J.E., 2005. Neurovascular coupling investigated with two-dimensional optical imaging spectroscopy in rat whisker barrel cortex. Eur J Neurosci 22, 1655-1666.

Blockley, N.P., Driver, I.D., Fisher, J.A., Francis, S.T., Gowland, P.A., 2012. Measuring venous blood volume changes during activation using hyperoxia. Neuroimage 59, 3266-3274.

Bohraus, Y., Goense, J., Logothetis, N.K., 2013. Decreased cerebral blood volume and flow in areas with negative BOLD indicates the mechanism for negative BOLD may be stimulus- and area-specific. In Proceedings of the 21st Annual Meeting of ISMRM, Salt Lake City, Utah, USA. p. 2341.

Boorman, L., Kennerley, A.J., Johnston, D., Jones, M., Zheng, Y., Redgrave, P., Berwick, J., 2010. Negative blood oxygen level dependence in the rat: a model for investigating the role of suppression in neurovascular coupling. J Neurosci 30, 4285-4294.

Boulant, N., Mangin, J.F., Amadon, A., 2009. Counteracting radio frequency inhomogeneity in the human brain at 7 Tesla using strongly modulating pulses. Magn Reson Med 61, 1165-1172.

Buxton, R.B., 2010. Interpreting oxygenation-based neuroimaging signals: the importance and the challenge of understanding brain oxygen metabolism. Frontiers in Neuroenergetics 2, 1-16.

Buxton, R.B., Wong, E.C., Frank, L.R., 1998. Dynamics of blood flow and oxygenation changes during brain activation: the balloon model. Magn. Reson. Med. 39, 855-864.

Chen, J.J., Pike, G.B., 2010. MRI measurement of the BOLD-specific flow-volume relationship during hypercapnia and hypocapnia in humans Neuroimage 53, 383-391.

Cohalan, C., Chen, J.J., Pike, G.B., 2009. Neuronal activity-induced cerebral blood volume changes in humans: mersurements with VASO and VERVE. In Proceedings of the 17th Annual Meeting of ISMRM, Hawai, USA. p. 13.

Donahue, M.J., Blicher, J.U., Ostergaard, L., Feinberg, D.A., Maclntosh, B.J., Miller, K.L., Günther, M., Jezzard, P., 2009. Cerebral blood flow, blood volume, and oxygen metabolism dynamics in human 
visual and motor cortex as measured by whole-brain multi-modal magnetic resonance imaging Journal of Cerebral Blood Flow and Metabolism 29, 1856-1866.

Donahue, M.J., Hoogduin, H., van Zijl, P.C., Jezzard, P., Luijten, P.R., Hendrikse, J., 2011. Blood oxygenation level-dependent (BOLD) total and extravascular signal changes and DeltaR2* in human visual cortex at 1.5, 3.0 and 7.0 T. NMR Biomed. 24, 25-34.

Duvernoy, H.M., Delon, S., Vannson, J.L., 1981. Cortical blood vessels of the human brain. Brain Res Bull 7, 519-579.

Galassi, M., Gough, B., 2006. GNU scientific library: reference manual. Network Theory Limited Bristol, UK.

Goense, J., Logothetis, N.K., Merkle, H., 2010. Flexible, phase-matched, linear receive arrays for highfield MRI in monkeys. Magn Reson Imaging 28, 1183-1191.

Goense, J., Merkle, H., Logothetis, N.K., 2012. High-resolution fMRI reveals laminar differences in neurovascular coupling between positive and negative BOLD responses. Neuron 76, 629-639.

Goense, J.B., Logothetis, N.K., 2008. Neurophysiology of the BOLD fMRI signal in awake monkeys. Curr Biol 18, 631-640.

Gray, L.H., Steadman, J.M., 1964. Determination of the oxyhaemoglobin dissociation curves for mouse and rat blood. J Physiol 175, 161-171.

Harel, N., Lee, S.P., Nagaoka, T., Kim, D.S., Kim, S.G., 2002. Origin of negative blood oxygenation level-dependent fMRI signals. J Cereb Blood Flow Metab 22, 908-917.

Hillman, E.M.C., Devor, A., Bouchard, M.B., Dunn, A.K., Krauss, G.W., Skoch, J., Bacskai, B.J., Dale, A.M., Boas, D.A., 2007. Depth-resolved optical imaging and microscopy of vascular compartment dynamics during somatosensory stimulation. Neuroimage 35, 89-104.

Hua, J., Jones, C.K., Qin, Q., van Zijl, P.C., 2013. Implementation of vascular-space-occupancy MRI at 7T. Magn Reson Med 69, 1003-1013.

Huber, L., Ivanov, D., Krieger, S.N., Gauthier, C., Roggenhofer, E., Henseler, I., Turner, R., Möller, H.E., 2013a. Measurements of cerebral blood volume and BOLD signal during hypercapnia and functional stimulation in humans at 7T: application to calibrated BOLD. In Proceedings of the 21st Annual Meeting of ISMRM, Salt Lake Citiy, Utah, USA. p 3339.

Huber, L., Ivanov, D., Krieger, S.N., Streicher, M.N., Mildner, T., Poser, B.A., Möller, H.E., Turner, R., 2013b. Slab-selective, BOLD-corrected VASO at 7 tesla provides measures of cerebral blood volume reactivity with high signal-to-noise-ratio. Magn Reson Med. doi: 10.1002/mrm.24916.

Hurley, A.C., Al-Radaideh, A., Bai, L., Aickelin, U., Coxon, R., Glover, P., Gowland, P.A., 2010. Tailored RF pulse for magnetization inversion at ultrahigh field. Magn. Reson. Med. 63, 51-58.

Hutchison, J.L., Lu, H., Rypma, B., 2013. Neural mechanisms of age-related slowing: the DeltaCBF/DeltaCMRO2 ratio mediates age-differences in BOLD signal and human performance. Cereb Cortex 23, 2337-2346.

Ivanov, D., Schäfer, A., Deistung, A., Streicher, M.N., Kabisch, S., Henseler, I., Roggenhofer, E., Jochimsen, T.H., Schweser, F., Reichenbach, J.R., Uludag, K., Turner, R., 2013. In vivo estimation of the transverse relaxation time dependence of blood on oxygenation at 7 tesla. In Proceedings of the 21st Annual Meeting of ISMRM, Salt Lake City, Utah, USA. p. 2472.

Jiang, J., Zhu, W., Shi, F., Liu, Y., Li, J., Qin, W., Li, K., Yu, C., Jiang, T., 2009. Thick visual cortex in the early blind. J Neurosci 29, 2205-2211.

Jin, T., Kim, S.G., 2008a. Cortical layer-dependent dynamic blood oxygenation, cerebral blood flow and cerebral blood volume responses during visual stimulation. Neuroimage 43, 1-9.

Jin, T., Kim, S.G., 2008b. Improved cortical-layer specificity of vascular space occupancy fMRI with slab inversion relative to spin-echo BOLD at 9.4 T. Neuroimage 40, 59-67.

Jochimsen, T.H., von Mengershausen, M., 2004. ODIN-object-oriented development interface for NMR. J Magn. Reson. 170, 67-78

Keller, A.L., Schuz, A., Logothetis, N.K., Weber, B., 2011. Vascularization of cytochrome oxidase-rich blobs in the primary visual cortex of squirrel and macaque monkeys. J Neurosci 31, 1246-1253. 
Kennerley, A.J., Berwick, J., Martindale, J., Johnston, D., Papadakis, N., Mayhew, J.E., 2005. Concurrent $\mathrm{fMRI}$ and optical measures for the investigation of the hemodynamic response function. Magn Reson Med 54, 354-365.

Kennerley, A.J., Harris, S., Bruyns-Haylett, M., Boorman, L., Zheng, Y., Jones, M., Berwick, J., 2012a. Early and late stimulus-evoked cortical hemodynamic responses provide insight into the neurogenic nature of neurovascular coupling. J Cereb Blood Flow Metab 32, 468-480.

Kennerley, A.J., Mayhew, J.E., Boorman, L., Zheng, Y., Berwick, J., 2012b. Is optical imaging spectroscopy a viable measurement technique for the investigation of the negative BOLD phenomenon? A concurrent optical imaging spectroscopy and fMRI study at high field $(7 \mathrm{~T})$. Neuroimage 61, 10-20.

Kim, S.G., 1995. Quantification of relative cerebral bloob-flow change by flow-sensitive alternating inversion-recovery (FAIR) technique - application to functional mapping. Magnetic Resonance in Medicine 34, 293-301.

Kim, S.G., Harel, N., Jin, T., Kim, T., Lee, P., Zhao, F., 2013. Cerebral blood volume MRI with intravascular superparamagnetic iron oxide nanoparticles. NMR Biomed 26, 949-962.

Kim, S.G., Ogawa, S., 2012. Biophysical and physiological origins of blood oxygenation leveldependent fMRI signals. J Cereb Blood Flow Metab 32, 1188-1206.

Kim, T., Hendrich, K.S., Masamoto, K., Kim, S.G., 2007. Arterial versus total blood volume changes during neural activity-induced cerebral blood flow change: implication for BOLD fMRI. J Cereb Blood Flow Metab 27, 1235-1247.

Kim, T., Kim, S.G., 2005. Quantification of cerebral arterial blood volume and cerebral blood flow using MRI with modulation of tissue and vessel (MOTIVE) signals. Magn Reson Med 54, 333-342.

Kim, T., Kim, S.G., 2011. Temporal dynamics and spatial specificity of arterial and venous blood volume changes during visual stimulation: implication for BOLD quantification. J Cereb Blood Flow Metab 31, 1211-1222.

Lee, S.P., Duong, T.Q., Yang, G., ladecola, C., Kim, S.G., 2001. Relative changes of cerebral arterial and venous blood volumes during increased cerebral blood flow: implications for BOLD fMRI. Magn. Reson. Med. 45, 791-800.

Liu, Y., Shen, H., Zhou, Z., Hu, D., 2011. Sustained negative BOLD response in human fMRI finger tapping task. PLoS One 6, e23839.

Logothetis, N.K., Augath, M., Murayama, Y., Rauch, A., Sultan, F., Goense, J., Oeltermann, A., Merkle, H., 2010. The effects of electrical microstimulation on cortical signal propagation. Nat Neurosci 13, 1283-1291.

Logothetis, N.K., Guggenberger, H., Peled, S., Pauls, J., 1999. Functional imaging of the monkey brain. Nat Neurosci 2, 555-562.

Lu, H., Golay, X., Pekar, J.J., van Zijl, P.C.M., 2003. Functional magnetic resonance imaging based on changes in vascular space occupancy. Magn. Reson. Med. 50, 263-274.

Lu, H., Hua, J., van Zijl, P.C., 2013. Noninvasive functional imaging of cerebral blood volume with vascular-space-occupancy (VASO) MRI. NMR Biomed 26, 932-948.

Lu, H., Zhao, C., Ge, Y., Lewis-Amezcua, K., 2008. Baseline blood oxygenation modulates response amplitude: Physiologic basis for intersubject variations in functional MRI signals. Magn. Reson. Med. 60, 364-372.

Mandeville, J.B., Marota, J.J., Kosofsky, B.E., Keltner, J.R., Weissleder, R., Rosen, B.R., Weisskoff, R.M., 1998. Dynamic functional imaging of relative cerebral blood volume during rat forepaw stimulation. Magnetic Resonance in Medicine 39, 615-624.

Martin, C., Martindale, J., Berwick, J., Mayhew, J., 2006. Investigating neural-hemodynamic coupling and the hemodynamic response function in the awake rat. Neuroimage 32, 33-48.

Martindale, J., Kennerley, A.J., Johnston, D., Zheng, Y., Mayhew, J.E., 2008. Theory and generalization of Monte Carlo models of the BOLD signal source. Magn. Reson. Med. 59, 607-618.

Moradi, F., Buracas, G.T., Buxton, R.B., 2012. Attention strongly increases oxygen metabolic response to stimulus in primary visual cortex. Neuroimage 59, 601-607. 
Mullinger, K.J., Mayhew, S.D., Bagshaw, A.P., Bowtell, R., Francis, S.T., 2014. Evidence that the negative BOLD response is neuronal in origin: a simultaneous EEG-BOLD-CBF study in humans. Neuroimage. doi: 10.1016/j.neuroimage.2014.02.029.

Pasley, B.N., Inglis, B.A., Freeman, R.D., 2007. Analysis of oxygen metabolism implies a neural origin for the negative BOLD response in human visual cortex. Neuroimage 36, 269-276.

Payne, S.J., 2006. A model of the interaction between autoregulation and neural activation in the brain. Math Biosci 204, 260-281.

Pfeuffer, J., Merkle, H., Beyerlein, M., Steudel, T., Logothetis, N.K., 2004. Anatomical and functional MR imaging in the macaque monkey using a vertical large-bore 7 Tesla setup. Magn Reson Imaging 22, 1343-1359.

Raz, A., Lieber, B., Soliman, F., Buhle, J., Posner, J., Peterson, B.S., Posner, M.I., 2005. Ecological nuances in functional magnetic resonance imaging (fMRI): psychological stressors, posture, and hydrostatics. Neuroimage 25, 1-7.

Schäfer, K., Blankenburg, F., Kupers, R., Grüner, J.M., Law, I., Lauritzen, M., Larsson, H.B., 2012. Negative BOLD signal changes in ipsilateral primary somatosensory cortex are associated with perfusion decreases and behavioral evidence for functional inhibition. Neuroimage 59, 3119-3127.

Schridde, U., Khubchandani, M., Motelow, J.E., Sanganahalli, B.G., Hyder, F., Blumenfeld, H., 2008. Negative BOLD with large increases in neuronal activity. Cereb Cortex 18, 1814-1827.

Shih, Y.Y., Chen, C.C., Shyu, B.C., Lin, Z.J., Chiang, Y.C., Jaw, F.S., Chen, Y.Y., Chang, C., 2009. A new scenario for negative functional magnetic resonance imaging signals: endogenous neurotransmission. J Neurosci 29, 3036-3044.

Shin, W., Geng, X., Gu, H., Zhan, W., Zou, Q., Yang, Y., 2010. Automated brain tissue segmentation based on fractional signal mapping from inversion recovery Look-Locker acquisition. Neuroimage 52, 1347-1354.

Shmuel, A., Augath, M., Oeltermann, A., Logothetis, N.K., 2006. Negative functional MRI response correlates with decreases in neuronal activity in monkey visual area V1. Nat Neurosci 9, 569-577.

Shmuel, A., Yacoub, E., Pfeuffer, J., de Moortele, P.-F.V., Adriany, G., Hu, X., Ugurbil, K., 2002. Sustained negative BOLD, blood flow and oxygen onsumption response and its coupling to the positive response in the human brain. Neuron 36, 1195-1210.

Smirnakis, S.M., Schmid, M.C., Weber, B., Tolias, A.S., Augath, M., Logothetis, N.K., 2007. Spatial specificity of BOLD versus cerebral blood volume fMRI for mapping cortical organization. J Cereb Blood Flow Metab 27, 1248-1261.

Smith, A.T., Williams, A.L., Singh, K.D., 2004. Negative BOLD in the visual cortex: evidence against blood stealing. Hum Brain Mapp 21, 213-220.

Stefanovic, B., Pike, G.B., 2005. Venous refocusing for volume estimation: VERVE functional magnetic resonance imaging. Magnetic Resonance in Medicine 53, 339-347.

Stefanovic, B., Warnking, J.M., Pike, G.B., 2004. Hemodynamic and metabolic responses to neuronal inhibition. Neuroimage 22, 771-778.

Tajima, S., Watanabe, M., Imai, C., Ueno, K., Asamizuya, T., Sun, P., Tanaka, K., Cheng, K., 2010. Opposing effects of contextual surround in human early visual cortex revealed by functional magnetic resonance imaging with continuously modulated visual stimuli. J Neurosci 30, 3264-3270.

Takano, T., Tian, G.F., Peng, W., Lou, N., Libionka, W., Han, X., Nedergaard, M., 2006. Astrocytemediated control of cerebral blood flow. Nat Neurosci 9, 260-267.

Turner, R., 2002. How much cortex can a vein drain? downstrean milustion of activation-related cerebral blood oxygenation changes. Neuroimage 16, 1062-1067.

Vafaee, M.S., Gjedde, A., 2004. Spatially dissociated flow-metabolism coupling in brain activation. Neuroimage 21, 507-515.

Wade, A.R., Rowland, J., 2010. Early suppressive mechanisms and the negative blood oxygenation level-dependent response in human visual cortex. J Neurosci 30, 5008-5019.

Weber, B., Keller, A.L., Reichold, J., Logothetis, N.K., 2008. The microvascular system of the striate and extrastriate visual cortex of the macaque. Cereb Cortex 18, 2318-2330. 
Wehrli, F.W., Rodgers, Z.B., Jain, V., Langham, M.C., Li, C., Licht, D.J., Magland, J., 2014. Timeresolved MRI oximetry for quantifying CMRO2 and vascular reactivity. Acad Radiol 21, 207-214.

Wong, E.C., Buxton, R.B., Frank, L.R., 1998. Quantitative imaging of perfusion using a single substraction (QUIPPS and QUIPSS II). Magnetic Resonance in Medicine 39, 702-708.

Woolsey, T.A., Rovainen, C.M., Cox, S.B., Henegar, M.H., Liang, G.E., Liu, D., Moskalenko, Y.E., Sui, J., Wei, L., 1996. Neuronal units linked to microvascular modules in cerebral cortex: response elements for imaging the brain. Cereb Cortex 6, 647-660.

Worsley, K.J., 2001. Statistical analysis of activation images. P. Jezzard and P. M. Matthews and S. M. Smith.

Xie, L., Kang, H., Xu, Q., Chen, M.J., Liao, Y., Thiyagarajan, M., O'Donnell, J., Christensen, D.J., Nicholson, C., Iliff, J.J., Takano, T., Deane, R., Nedergaard, M., 2013. Sleep drives metabolite clearance from the adult brain. Science 342, 373-377.

Yang, Y., Gu, H., Stein, E.A., 2004. Simultaneous MRI acquisition of blood volume, blood flow, and blood oxygenation information during brain activation. Magn Reson Med 52, 1407-1417.

Yaseen, M.A., Srinivasan, V.J., Sakadzic, S., Radhakrishnan, H., Gorczynska, I., Wu, W., Fujimoto, J.G., Boas, D.A., 2011. Microvascular oxygen tension and flow measurements in rodent cerebral cortex during baseline conditions and functional activation. J Cereb Blood Flow Metab 31, 1051-1063.

Zappe, A.C., Uludag, K., Logothetis, N.K., 2008. Direct measurement of oxygen extraction with fMRI using 6\% CO2 inhalation. Magn Reson Imaging 26, 961-967.

Zhao, F., Wang, P., Hendrich, K., Ugurbil, K., Kim, S.G., 2006. Cortical layer-dependent BOLD and CBV responses measured by spin-echo and gradient-echo fMRI: insights into hemodynamic regulation. Neuroimage 30, 1149-1160.

Zong, X., Kim, T., Kim, S.G., 2012. Contributions of dynamic venous blood volume versus oxygenation level changes to BOLD fMRI. Neuroimage 60, 2238-2246. 


\section{Figure captions:}

Fig. 1: Evaluation procedure for estimating BOLD and CBV-related signal changes. Multi-echo images with and without blood nulling are acquired during three different stimulation paradigms. Time series of BOLD and BOLD-corrected VASO were used to define ROIs of PBR and NBR (using FSL). Signal changes within these ROIs are considered with respect to PBR and NBR, with respect to 'arterial' and 'venous' CBV, and with respect to surface and deeper cortical laminae.

Fig 2: Statistical maps of the VASO and BOLD responses to three different stimulation paradigms in four subjects. ROls of positive and negative signal changes were consistent across subjects. ROls suggesting activation and deactivation were almost identical in VASO and BOLD, across subjects.

Fig. 3: Laminar-dependence of BOLD and VASO. Negative responses refer to ROIs with NBR during the small flickering checkerboard stimulation paradigm. Positive responses refer to the same ROls, but during the excitatory task of the full-field flickering checkerboard. A.) For the excitatory task, GE BOLD was significantly dominated by signal from the cortical surface, compared to deeper grey matter. The VASO signal on the other hand did not exhibit this surface dominance. B.) The BOLD signal lost its surface dominance for the inhibitory task and its signal change was significantly smaller at the surface compared to deeper layers. The VASO signal change was significantly dominated by signal from the surface vessels. C.) In voxels containing surface vessels, $82 \%$ of $\triangle C B V$ comes from oxygenated 'arterial' blood in the case of the positive response. In voxels without surface vessels, the amount of 'arterial' $\triangle C B V$ is only $63 \%$. D.) Discussion of the effect, when $C B V_{\text {rest }}$ distribution is assumed to be different in surface and deeper voxels. Based on the ratio of the voxel size and cortical thickness, $12 \%$ higher $\mathrm{CBV}_{\text {rest }}$ is assumed in surface voxels (e.g. surface/deeper $\mathrm{CBV}$ ratio of $\approx 0.56 / 0.44$ (Kennerley et al., 2005), or $\approx 0.57 / 0.43$ (Zhao et al., 2006)). These ratios are estimated based on the published profiles (Fig. 4D in (Kennerley et al., 2005) and Fig. 3A in (Zhao et al., 2006), respectively). When laminarindependent homogeneous baseline CBV is assumed, the baseline CBV in surface voxels is underestimated and thus, the relative CBV change calculated from VASO signal change is overestimated.

Fig. 4: $C B V$ and BOLD time courses in ROls of PBR and NBR for the stimulation paradigm using the small flickering checkerboard. Post-stimulus baseline crossing was significantly faster in ROls of NBR compared to ROIs of PBR for both CBV and BOLD signal changes.

Fig. 5: The 'arterial' and 'venous' composition of CBV changes. A.) VASO time courses for different TEs during full field flickering checkerboard stimulation. With longer TEs (TE > $12 \mathrm{~ms}$, black and grey) the BOLD corrected VASO signal change increases. Signal changes with shorter TE reflect both, 'venous' and 'arterial' CBV change. Signal changes with longer TE captures 'arterial' CBV change only. It can be seen, that signal change with shorter $T E$ has a significantly faster response compared to longer TE timecourses, indicating a faster 'arterial' response compared to the 'venous' one. The difference in these time courses is used to estimate the 'arterial' and 'venous' CBV changes individually (section 2.1.1 and appendix). Signal change of TE = $52 \mathrm{~ms}$ is depicted for sake of completeness, but was not used for the calculation of 'arterial' and 'venous' CBV due to its high noise level (appendix). Error bars refer to inter-subject standard deviation B.) Timecourses of total, 'arterial' and 'venous' CBV during an excitatory task. 'Venous' $\mathrm{CBV}_{\mathrm{v}}$ was significantly smaller than 'arterial' CBV and showed a delayed response. 'Arterial' $\mathrm{CBV}_{\mathrm{a}}$ showed a distinct post-stimulus undershoot. C.) Timecourses of total, 'arterial' and 'venous' CBV during an inhibitory task (small flickering checkerboard). Again, change in total $\mathrm{CBV}_{\mathrm{t}}$ was dominated by the 'arterial' component. Due to the high noise level, $\mathrm{CBV}_{a}$ and $\mathrm{CBV}_{\mathrm{v}}$ are depicted temporally filtered, overlaid on the unfiltered timecourses (grey). D.) Summary of blood volume changes during excitatory and inhibitory tasks. Error bars of 'arterial' and 'venous' CBV refer to standard deviation across trials.

Fig. 6: $C B F$ in response to the small flickering checkerboard stimulation paradigm. A.) baseline perfusion weighted image of one of the subjects. B.) statistical CBF activation map indicating a CBF increase and CBF 
decrease in regions of PBR and NBR in the same subject. C.) CBF time courses in ROIs of PBR and NBR averaged over four subjects.

Fig. 7: Timecourses of VASO CBV and BOLD signal during the rotating ring stimulation task. Timecourses are shown for surface and deeper GM voxels within these ROIs. During the excitatory task, the BOLD signal change is dominated by surface voxels while $\triangle \mathrm{CBV}$ shows comparable timecourses in surface and deeper laminae. During the inhibitory rotating ring stimulation paradigm, $\triangle C B V$ and BOLD signal time courses have a faster post-stimulus baseline crossing. BOLD signal loses its surface dominance and the $\triangle C B V$ seems to predominantly occur in the surface voxels.

Fig. 8: SS-SI-VASO functional activation in an anesthetized monkey acquired for comparison between the results obtained in humans and monkeys. The panels show the BOLD and VASO signal change in a sagittal slice through macaque striate cortex. The ROIs of PBR showed a VASO signal decrease. Green arrows point to the regions processing the rotating checkerboard pattern. NBR in areas processing the ring of grey background, $\left(3^{\circ}-9^{\circ}\right.$, middle part of ROI) and PBR regions correspond to the checkerboard ring $\left(9^{\circ}-11^{\circ}\right)$. ROIs of NBR show a small VASO signal decrease, indicating vasodilation consistent with previous studies. 
Figures:

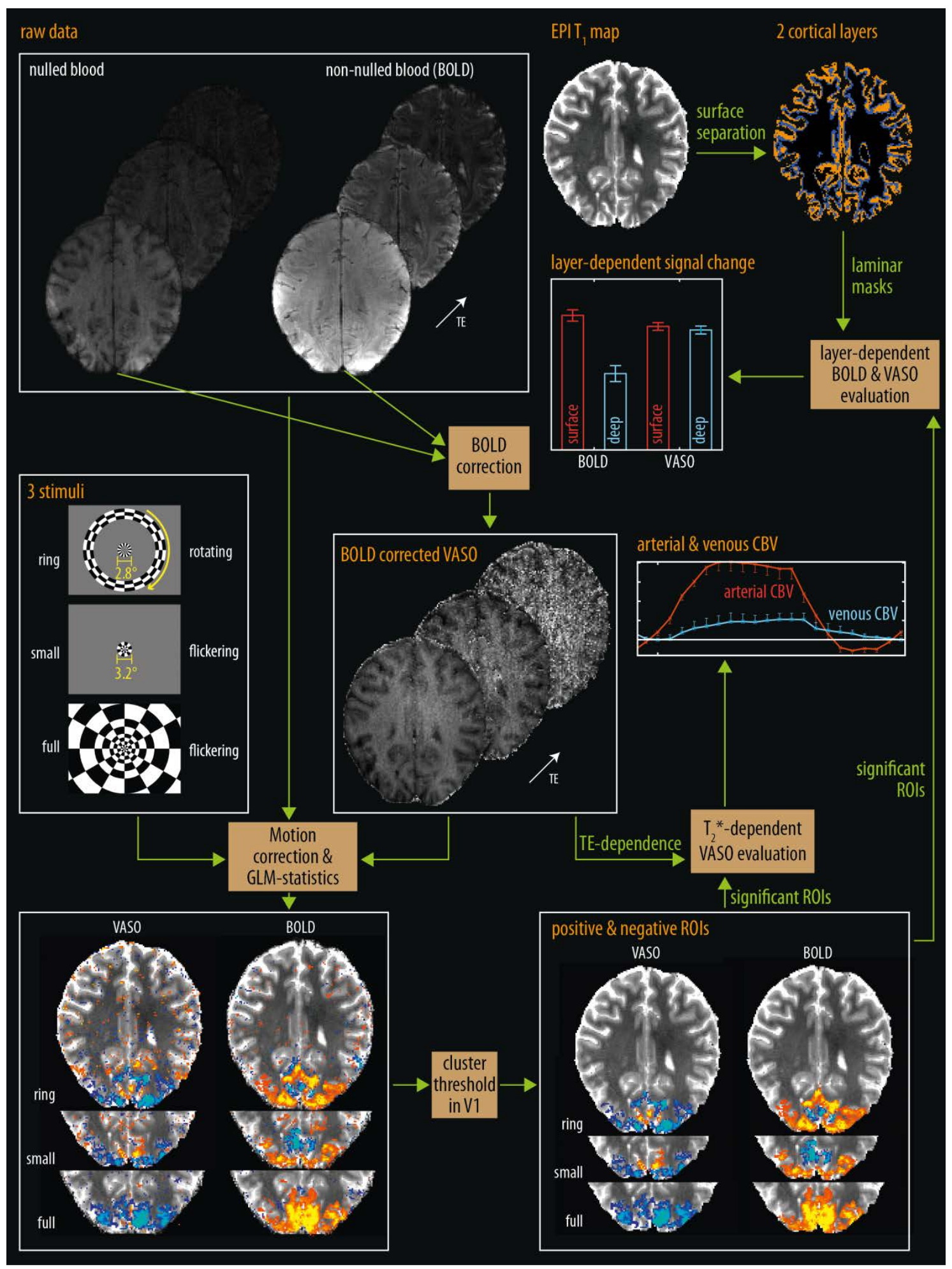

Fig 1. 


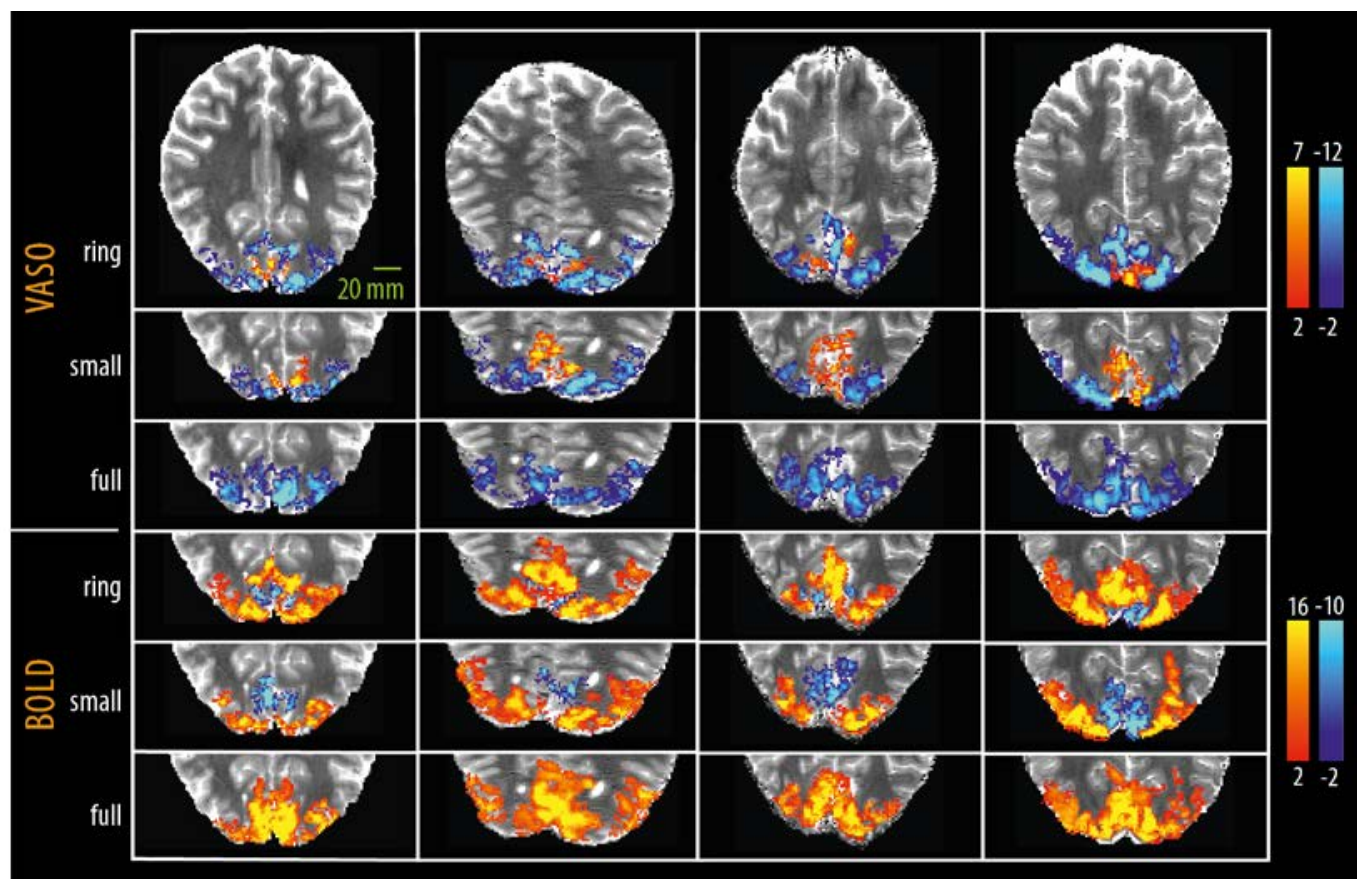

Fig 2. 

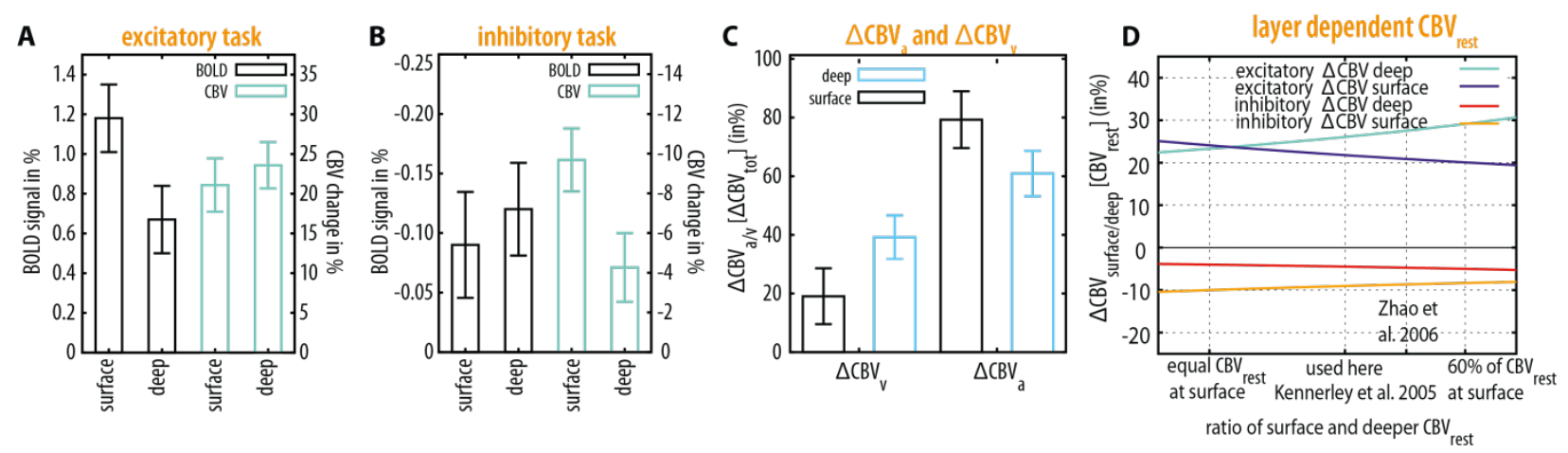

Fig 3. 

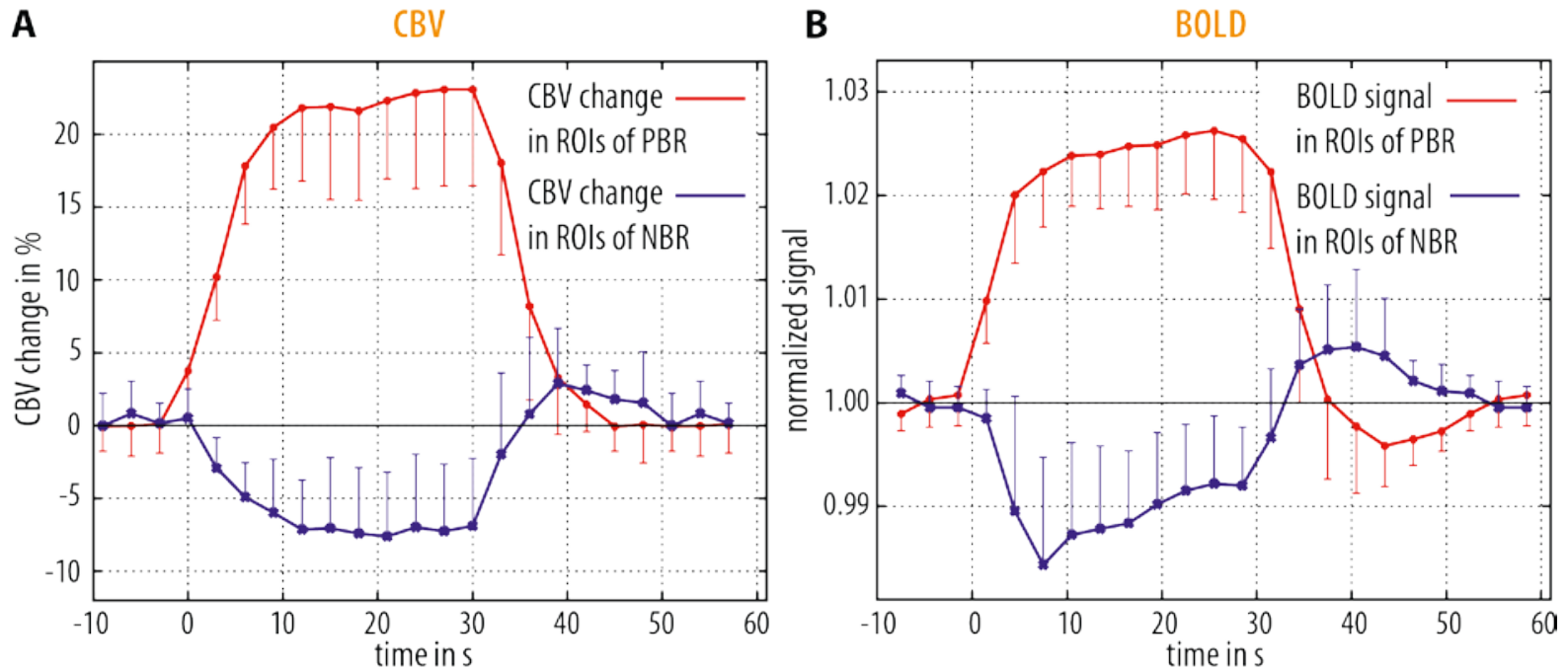

Fig 4. 

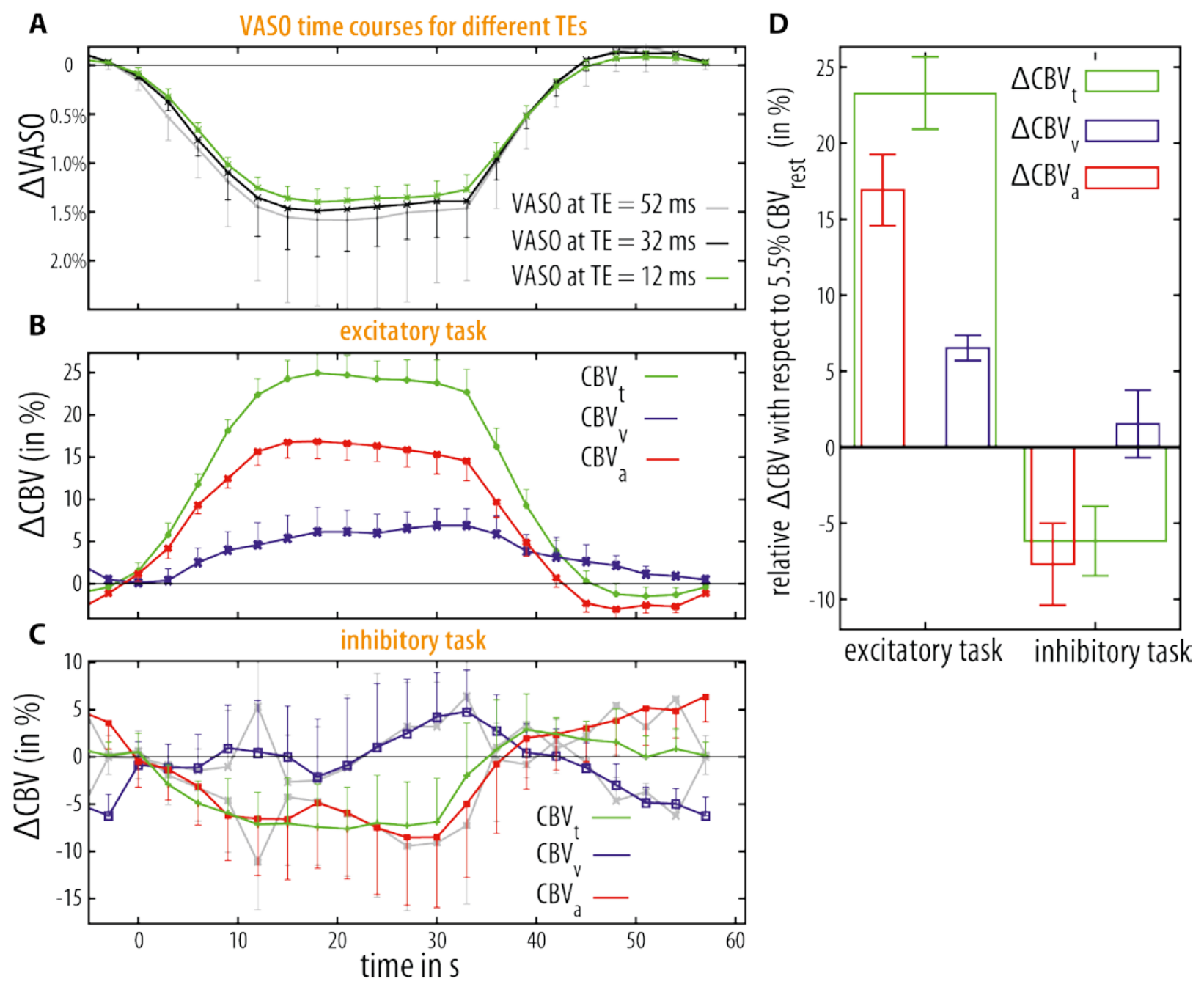

Fig 5. 

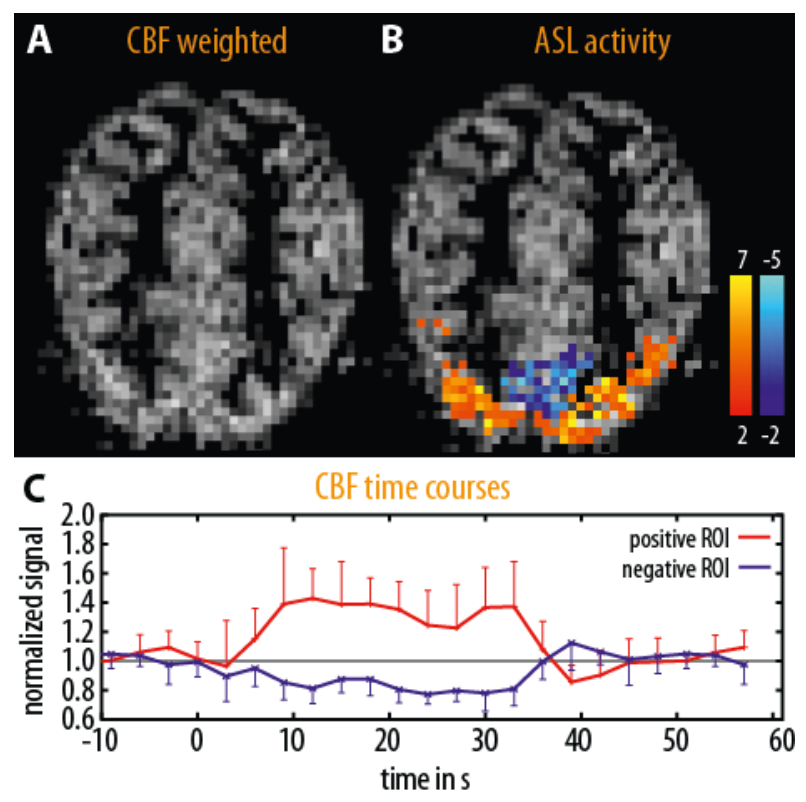

Fig 6 
excitatory
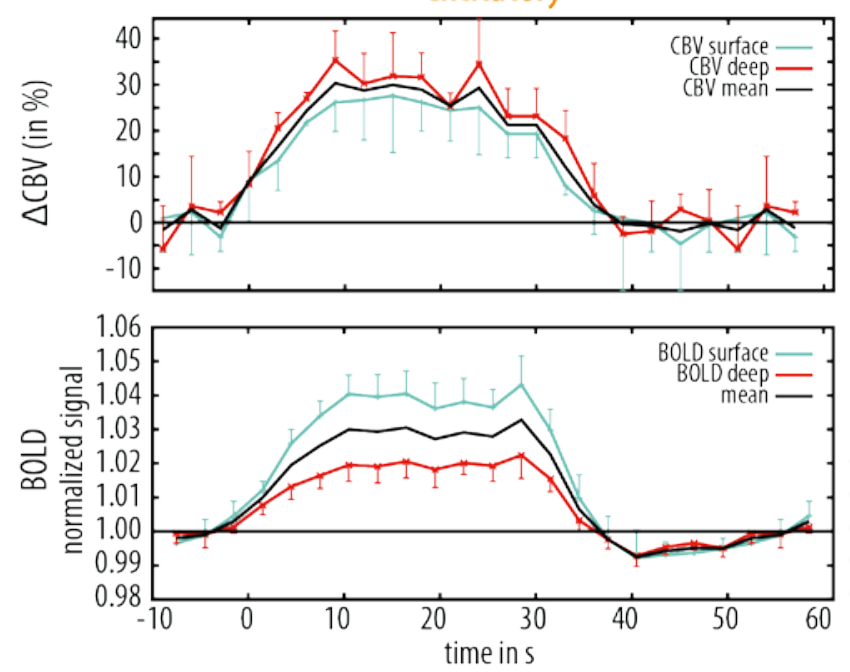

inhibitory
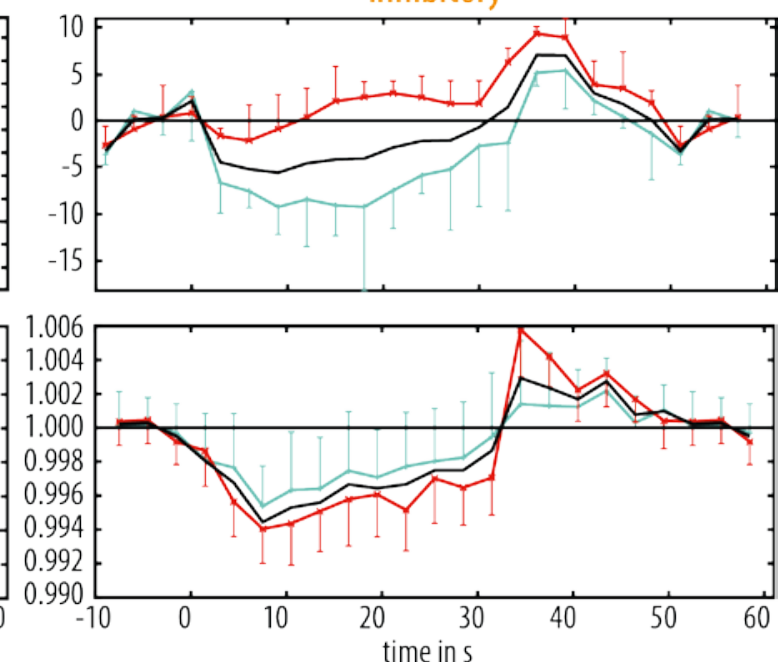

Fig. 7 


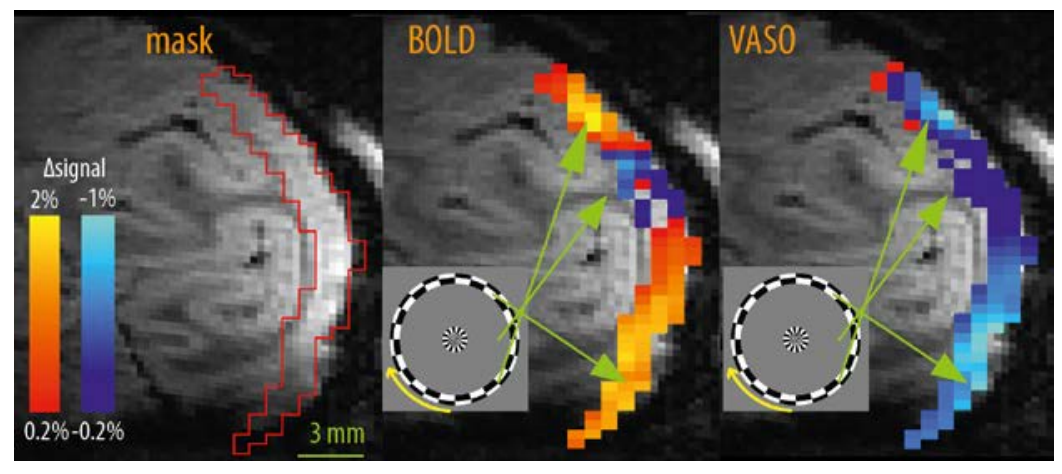

Fig 8 